This document was prepared in conjunction with work accomplished under Contract No. DE-AC09-96SR18500 with the U.S. Department of Energy.

This work was prepared under an agreement with and funded by the U.S. Government. Neither the U. S. Government or its employees, nor any of its contractors, subcontractors or their employees, makes any express or implied: 1 . warranty or assumes any legal liability for the accuracy, completeness, or for the use or results of such use of any information, product, or process disclosed; or 2 . representation that such use or results of such use would not infringe privately owned rights; or 3 . endorsement or recommendation of any specifically identified commercial product, process, or service. Any views and opinions of authors expressed in this work do not necessarily state or reflect those of the United States Government, or its contractors, or subcontractors. 
WSRC-MS-2006-0422 Rev. 1

\title{
Accumulation of Radiocesium by Mushrooms in the Environment: A Literature Review
}

\author{
Martine C. Duff and Mary Lou Ramsey \\ Savannah River National Laboratory \\ Bldg. 773-43A, Rm. 217 \\ Savannah River Site \\ Aiken, SC 29808 \\ e-mail: martine.duff@srnl.doe.gov
}

\begin{abstract}
During the last 50 years, a large amount of information on radionuclide accumulators or "sentinel-type" organisms in the environment has been published. Much of this work focused on the risks of food-chain transfer of radionuclides to higher organisms such as reindeer and man. However, until the 1980's and 1990's, there has been little published data on the radiocesium $\left({ }^{134} \mathrm{Cs}\right.$ and ${ }^{137} \mathrm{Cs}$ ) accumulation by mushrooms. This presentation will consist of a review of the published data for ${ }^{134,137} \mathrm{Cs}$ accumulation by mushrooms in nature. This review will discuss the aspects that promote ${ }^{134,137} \mathrm{Cs}$ uptake by mushrooms and focus on mushrooms that demonstrate a large propensity for use in the environmental biomonitoring of radiocesium contamination. It will also provide descriptions of habitats for many of these mushrooms and discuss on how growth media and other conditions relate to $\mathrm{Cs}$ accumulation.
\end{abstract}

Keywords: Concentration ratio; Transfer factor; Aggregate transfer factor; Biomonitoring; Chernobyl

\section{Introduction}

Radiocesium (primarily ${ }^{137} \mathrm{Cs}$ and ${ }^{134} \mathrm{Cs}$ isotopes) is produced mainly by the fission of nuclear fuel. Releases of ${ }^{134,137} \mathrm{Cs}$ into the environment have occurred primarily from nuclear weapons testing, nuclear fuel reprocessing and nuclear reactor accidents like that of Chernobyl in 1986. Because these processes release ${ }^{134,137} \mathrm{Cs}$ as well as other radionuclides into the environment, there has been considerable concern about the transfer of radionuclides to higher organisms in the food chain.

One of the common approaches to assess the food chain transfer of radionuclides is the radiometric measurement of radionuclides that are accumulated by organisms that comprise the lower portion of the food chain. In some cases, when these lower organisms (such as wild mushrooms) have a large affinity for radionuclides, the consumption of these organisms by humans can pose a considerable health concern from a radiological dose perspective (Grueter, 1964). Despite this realization, there is fairly limited biomonitoring data on radionuclide levels in mushrooms today. Baeza and co-workers (2004) and many other researchers that are cited in this review concur with this observation but they note that considerably more data are available for ${ }^{137} \mathrm{Cs}$ than for other ${ }^{134} \mathrm{Cs}$, alpha- and betaemitting radionuclides. 
WSRC-MS-2006-0422 Rev. 1

\section{Background on Mushrooms and Conditions that Influence Radiocesium Accumulation}

There are $\sim 13,500$ species of mushrooms and generally, three main groups of mushrooms: gilled, non-gilled, and puffballs and their relatives. Those mushrooms of interest as radiocesium accumulators are typically gilled or have pores or spines under their caps and they have stalks. Typical names for these mushrooms have the following words in them: roof, sheath, and common meadow (mushrooms); milk, crumble, death, waxy, web, and inky caps; tricholomas; ringstalks; mottle, pink, and brittle gills; and various "tooths". The common names for Cs-accumulating mushrooms are listed next to their Latin names as listed in Table 1.

Mushrooms typically prefer forests and grasslands. However, almost any ecosystem (including deserts and tundra) with the right substrates will support their growth.

Mushrooms often require a particular growth medium to live in. The media may consist of a decaying portion of a tree, open grasslands, animal manure or areas directly under a tree in leaf or needle litter. Growth media for mushrooms has been defined using the following variables: wood, litter layer, $0-5 \mathrm{~cm}$ soil profile and $>5 \mathrm{~cm}$ soil profile by Yoshida and researchers (1994). Kammerer et al. (1994) divided mushrooms according to their living habitats. The first group was the saprophytes, which live on and metabolically consume organic matter. The second group was the parasitic mushrooms, which live on other species in a non-symbiotic relationship. The third group was the symbiotic species, which through the use of underground mycorrihiza, form a close relationship with their host plant.

In nature mushrooms can be confused with manure, seed pods, bone, lichens, some flowering plants that are not green in color, insect sacs or galls, and rotten wood. When removed from their growth medium, mushrooms can break under their own weight. Due to their fragile structure and high water content, even gentle handling is likely to make the recognition by species difficult after collection. Photography of the mushroom prior to removal from its growth habit is highly recommended in these cases. Their above ground portions or fruiting bodies (which are used for reproduction through spore release) usually appear between spring and early winter and their emergence is typically correlated with recent rainfall.

There are several field guides, which we used to obtain general information about mushrooms as mentioned above such as Læssøe (2002), McKnight and McKnight (1987), and Roody (2003). These guides have detailed descriptions about the identification of specific species of mushrooms and the locations that they can be found in. A species identification of mushrooms can be very difficult due to similarities between various types of species. Many mushrooms are poisonous. Consumption of mushrooms is not recommended if one lacks the expertise to distinguish the poisonous ones from those that are not.

Uptake of radiocesium by basidiomycete mushrooms was observed as early as the 1960s by $\mathrm{H}$. Grueter (1964; 1971). In these studies, mushrooms from sandy pine needlecovered soils had higher ${ }^{137} \mathrm{Cs}$ levels than those from soils in deciduous woods and meadows. The relatively high degree of Cs uptake was attributed to the sandy soil having a lower competition for ${ }^{137} \mathrm{Cs}$ (through sorption) than that of the soils in meadows and deciduous forests, which have a mineral composition that absorbs Cs more strongly. Additionally, organic material in soil O-horizons has a lower affinity for Cs than mineral soils and therefore, mushrooms that grow in organic soils are likely to have higher levels 
of Cs than those that grow in mineral soils (Kammerer et al., 1994). Kammerer and researchers (1994) also observed a higher average ${ }^{137} \mathrm{Cs}$ concentration in mushrooms that are symbiotic than those that are saprophytic and parasitic. However, the range in values for ${ }^{137} \mathrm{Cs}$ in these groups was the largest for the saprophytic species.

The depth at which underground portions of the mushrooms inhabit in the soil is also an important factor. Yoshida and researchers (1994) concluded that highest average concentration of ${ }^{137} \mathrm{Cs}$ was for those mushrooms that inhabited the surface soil $(0$ to $5 \mathrm{~cm})$ layer (with mycelia being noticeably present at that depth). They and Kammerer et al. (1994) attribute Cs uptake to be related to individual species or groups thereof-with saprophytes having less accumulation than those mushrooms living on the uppermost portion of the soil. Additionally, the ratios of ${ }^{137} \mathrm{Cs}$ for above and below ground portions of mushrooms from conifer forests typically exceed those ratios for mushrooms in deciduous forests (Vinichuk and Johanson, 2003 and references therein).

Mushrooms from higher altitudes can have higher radiocesium levels than those at lower altitudes (Heinrich, 1993). However, the soil properties of the soils in these studies may have a greater influence on radiocesium uptake than altitude as discussed by Heinrich. This was because the soil at the higher altitude was high in humus and more acidic. Such conditions favor a greater amount of plant-available Cs percentage than that of the other soils at lower elevations.

Depth and soil horizon can also have an influence on the ratio of ${ }^{134} \mathrm{Cs}$ to ${ }^{137} \mathrm{Cs}$ in mushrooms (Yoshida et al., 1994; Kammerer et al., 1994). This was attributed to periods of time when the aerial deposition of ${ }^{134} \mathrm{Cs}$ relative to ${ }^{137} \mathrm{C}$ varied greatly (such as from Chernobyl) and the soil at greater depths did not reflect the higher levels of ${ }^{134} \mathrm{Cs}$ relative to ${ }^{137} \mathrm{C}$ because the migration of ${ }^{134} \mathrm{Cs}$ in the soil column may be slow (Oolbekkink and Kuyper, 1989 and references therein). The levels (and ratios) of ${ }^{134} \mathrm{Cs}$ and ${ }^{137} \mathrm{Cs}$ may also vary (from that of the original source term) in mushrooms because ${ }^{134} \mathrm{Cs}$ can be discriminated from ${ }^{137} \mathrm{Cs}$ by some fungal species (Guillitte et al. 1987).

Other factors in addition to growth medium characteristics such as rainfall, soil (stable) Cs and $\mathrm{K}$ concentrations, climate, soil $\mathrm{pH}$, time of sampling, Cs source term, and growth substrate (such as rotting wood) may influence Cs uptake (as previously discussed; Oolbekkink and Kuyper, 1989 and references therein; Heinrich 1993), but most report that soil factors or conditions, such as $\mathrm{pH}$ and organic matter content and preferred growth medium probably have a great affect on the accumulation of $\mathrm{Cs}$ as well as other radionuclides by mushrooms (Battison et al., 1989). Finally, there are clearly several species of fungi that have a unique ability to accumulate Cs under a variety of conditions as will be discussed in this presentation.

\section{Approaches to Comparing Accumulation Values}

There are several approaches used to evaluate and compare the relative affinity that mushrooms have for radiocesium. We will discuss the assumptions that were made with these methods and the impact of making these assumptions.

The ratio of the Cs concentration in the mushroom (or fruiting body in $\mathrm{Bq} \mathrm{Cs} / \mathrm{kg} \mathrm{dw}$ ) relative to that of its growth medium (i.e., soil or rotting wood, etc. in $\mathrm{Bq} \mathrm{Cs} / \mathrm{kg} \mathrm{dw}$ ) is called a transfer factor (TF) or concentration ratio (CR). The TF is a metric that is used to evaluate the relative accumulation of $\mathrm{Cs}$ for various mushroom species. 
Toal et al. (2002) report the uptake of ${ }^{137} \mathrm{Cs}$ relative to the amount that is deposited by aerial deposition on the soil or the total soil ${ }^{137} \mathrm{Cs}$ inventory with the use of aggregate transfer factor or $\mathrm{T}_{\text {ag }}$ values. These $\mathrm{T}_{\mathrm{ag}}$ values (in $\mathrm{m}^{2} / \mathrm{kg} \mathrm{dw}$ ) are based on the amount of ${ }^{137} \mathrm{Cs}$ in the mushroom (in $\mathrm{Bq} / \mathrm{kg} \mathrm{dw}$ ) relative to the ${ }^{137} \mathrm{Cs}$ content in the aerial deposition (in $\mathrm{Bq} / \mathrm{m}^{2} \mathrm{dw}$ ). In their study, Toal and researchers cite literature data that indicates that all of the (aerially deposited) soil ${ }^{137} \mathrm{Cs}$ is in the upper $20 \mathrm{~cm}$ of the soil profile.

Oolbekkink and Kuyper (1989) discuss their preference for using TF values that are based on soil radiocesium levels as opposed to $\left(\mathrm{T}_{\mathrm{ag}}\right)$ values that are based on aerial deposition because the uptake of radiocesium by mushrooms occurs through their growth medium and not directly from the air. The use of $\mathrm{T}_{\text {ag }}$ values (as used in the later work by Toal et al., 2002) may be more applicable for the study of Cs uptake by lichens and mosses, which remove Cs directly from aerial fallout and do not have roots to remove soil Cs. However, the use of TF values requires one to assume choose a soil depth (for radiocesium activity concentration determination) at which the mushroom resides for radiocesium analysis. Toal and researchers recognize that comparisons based on ${ }^{137} \mathrm{Cs}$ inventory may not be practical and that knowing the inventory within regions of the profile that pertain to where the mushrooms reside may give a better approach for comparison.

Work by Vinichuk and Johanson (2003) shows that the total ${ }^{137} \mathrm{Cs}$ activity concentration in the soil and the fungal mycelium concentration can vary with depth, fungal type, and also with the selected increments of sampled depth (e.g., 1 or $2 \mathrm{~cm}$ intervals). This observation used TF values that were based on soil activity concentration (at 1 and $2 \mathrm{~cm}$ intervals) within this particular study. If we were to consider the impact of these observations, we find it somewhat difficult to compare the TF values obtained from one study with that of another. This is because the uptake of Cs could vary with depth because of differences in soil properties also vary with depth (such as soil organic matter content) as discussed by Kammerer et al. (1994), the density of fungal mycelium, and competition from other living species. Most all of the references that we reviewed noted the problems with estimating fungal mycelium as well as the process of generalizing Cs accumulation.

Other researchers have ratioed the radiocesium activity concentrations of the mushrooms that they analyzed to that of one species (Xerocomus badius also known as Boletus badius) which was common to most all of their sampling areas (as in Kammerer et al., 1994). This allowed these researchers to normalize their data to the radiocesium of one species. These researchers utilized TF values (based on $\mathrm{Bq} / \mathrm{kg}$ fw to $\mathrm{Bq} / \mathrm{kg}$ dw in the organic or O-horizon) as well.

A compilation of data on the accumulation of Cs by mushrooms will be presented in this review. We will also present published data on approaches for comparing radiocesium uptake amongst mushroom types and sampled regions as well as discuss the importance of growth media characteristics on radiocesium accumulation.

\section{Mushrooms with a Moderate to High Affinity for Radiocesium}

Data for the mushrooms that have been observed to accumulate high levels of Cs are listed in Table 1. The mushroom species represented here were collected in Europe, Japan and North America. Most all of the data in this table represents the above ground portions of the fungi unless otherwise noted. 
Studies have also observed radiocesium in the below ground portions of fungi (Vinichuk and Johanson, 2003) and the ${ }^{137} \mathrm{Cs}$ levels tend to be lower in the buried fungal mycelium than in the above ground fruiting bodies. Haselwandter (1978), Heinrich (1993) noted that portions of the caps or fruiting bodies (stems plus caps) tend to be rich in ${ }^{137} \mathrm{Cs}$ relative to their underground fungal masses. Vinichuk and Johanson (2003) noted that symbiotic fungi have high metabolic rates. As noted by Haselwandter and Berreck (1994), a study by Clint et al. (1991) observed that Cs is complexed by cap pigments in some boletes. This might explain why high uptake of $\mathrm{Cs}$ in the fruiting body, which includes the cap is observed. Heinrich (1993) determined the levels of ${ }^{137} \mathrm{C}$ in the lamella (gills), cap and stalk of Boletus edulis and observed that the gills had 2.5 times the levels of ${ }^{137} \mathrm{Cs}$ as the cap and the stalk. Radiography studies with Rozites caperata also showed higher levels of ${ }^{137} \mathrm{Cs}$ in the gills relative to the cap.

\section{$\underline{\text { Amanitas }}$}

Most Amanita sp. mushrooms are poisonous so they are not likely to be a concern with regard to human consumption, which is a driver for most studies on radionuclide accumulation by edible foods. Amanitas are mostly symbiotic and are typically found in deciduous and conifer forests. These gilled fungi have a moderate affinity for Cs as has been observed in several studies (Table 1). However, high uptake ${ }^{137} \mathrm{Cs}$ has been observed for Amanita rubescens which was growing in a spruce and fir forest that had a low cation exchange capacity, low pH and high humus content (Table 1; Heinrich, 1993). When cooked, Amanita rubescens is edible so there is some concern to man with regard to radiocesium uptake. Similar observations were made by Vinichuk and Johanson (2003) for Amanita muscaria (Table 1). In general, the reported TF values for the Amanitas in Table 1 are less or equal to 1.0 and the $T_{\text {ag }}$ value for Amanita rubescens is low relative to the other species in this table.

\section{$\underline{\text { Boletus }}$}

Boletes or Boletus mushrooms have a high affinity for Cs (Byrne, 1989; Heinrich, 1993; Kammerer et al., 1994; Toal et al., 2002; Vinichuk and Johanson, 2003; Řanda and Kučera, 2004)—see Table 1. Boletus and Suillus are two of the primary genuses that make up the family of Boletaceae. These two types of mushrooms as well as those of the genus Xerocomus often have common names that have "bolete" in them.

Boletus (or Suillus) variegatus prefer growing in sandy, acidic soils whereas Boletus edulis or porcini mushrooms prefer growth in mossy woodlands. Although some boletes are inedible or poisonous, many boletes are prized for their culinary value. Due to their marked ability to accumulate radiocesium, they pose some concern with regard to human consumption. Boletes are typically found in deciduous and conifer forests. Cesium-137 values range from a thousand $\mathrm{Bq} / \mathrm{kg}$ of ${ }^{137} \mathrm{Cs}$ to more than a hundred thousand $\mathrm{Bq} / \mathrm{kg}$ of ${ }^{137} \mathrm{Cs}$. High levels of ${ }^{137} \mathrm{Cs}$ uptake were also observed prior to the Chernobyl release in spring of 1986 (Byrne, 1988).

In contrast to the Amanitas, the reported TF values for the Boletes (Table 1) are as high as 5.0, which is a low value relative to TF values for other mushroom species in this table. In most cases, the high TF values that are observed for the Boletes (those with TF values greater than 1.0) have ${ }^{137} \mathrm{Cs}$ activity levels that meet or exceed $1000 \mathrm{~Bq}$ of ${ }^{137} \mathrm{Cs} / \mathrm{kg} \mathrm{dw}$ (or fw). Data reported for aggregate transfer values are reported in Table 1. Of those 
WSRC-MS-2006-0422 Rev. 1

reported values in Table 1, which range from 0.00007 to as high as 0.21 , the two reported $\mathrm{T}_{\mathrm{ag}}$ values of 0.0027 and 0.056 for the Boletes are relatively low.

\section{Cantharellus, Cortinarius and Clitocybe}

Like the boletes, many chanterelle or Cantharellus mushrooms are prized for their culinary value. These fungi, which grow on living or rotten wood have been observed to accumulate several tens of thousands $\mathrm{Bq} / \mathrm{kg}$ of ${ }^{137} \mathrm{Cs}$ - making their direct consumption by man a potential risk. Cantharellus cibarius and C. tubaeformis grow in groups in old spruce and pine forests. Cantharellus tubaeformis is also found in deciduous forests.

Cortinarius mushrooms such as Cortinarius (or Rozites) caperatus (as listed in Table 1) also have high affinities for Cs as noted by Byrne (1988), Kammerer et al. (1994) and others as noted in Table 1. Cortinarius mushrooms are gilled and saprotrophic species. Cortinarius armillatus are typically found in small groups in damp, organic-rich boggy woodlands whereas $C$. caperatus are found in small groups under conifers and deciduous trees like beech. Cortinarius caperatus have wrinkled or furrowed caps and they prefer acidic soils, which are likely to support Cs uptake as previously discussed.

Although they are not widely represented in the literature, the Clitocybe infundibuliformis (or C. gibba) mushroom can have ${ }^{137} \mathrm{Cs}$ and ${ }^{134} \mathrm{Cs}$ activity values as high as ten thousand $\mathrm{Bq} / \mathrm{kg}$ or more. These mushrooms also live on decayed material. The have low TF values $(<0.7)$ relative to the Cantharellus and Cortinarius mushrooms, which have TF values range from 1.0 to $>4.0$, with the exception of Cantharellus lutescens. No $\mathrm{T}_{\mathrm{ag}}$ values were reported as noted in Table 1.

Hebeloma sp., Hydnum repandum, Hygrophorus russula and Hypholoma fasciculare

Most Hebeloma sp. and Hypholoma fasciculare are poisonous (or inedible) mushrooms that are gilled. Depending on species, Hebeloma fungi have a variety of growth habits but they typically inhabit organic-rich material at the soil surface (Yoshida et al., 1994). This preference for organic growth media has been attributed to high Cs accumulation as previously discussed. Hypholoma fasciculare prefer growth on rotten deciduous tree stumps and roots.

Hygrophorus russula mushrooms grows in fairy ring formations, in groups or as scattered single mushrooms in oak forests. Hydnum repandum grows in groups or small clusters in conifer and deciduous woods. Unlike most Hebeloma sp. and Hypholoma fasciculare, the Hygrophorus russula and Hydnum repandum mushrooms are edible. These mushroom species have been observed to accumulate up to several thousand $\mathrm{Bq} / \mathrm{kg}$ of ${ }^{137} \mathrm{Cs}$ (see Table 1). The TF values for Hydnum repandum are high (typically 1 to $>4.0$ ) and they compare well with the ${ }^{137} \mathrm{Cs}$ activity levels for these mushrooms.

For Hypholoma fasciculare, the $\mathrm{T}_{\text {ag }}$ values were that were reported in Table 1 were close to that of the highest reported within the table ( $T_{\text {ag }}$ values of 0.09 to 0.1$)$. No $T_{\text {ag }}$ values were reported for Hebeloma sp., Hydnum repandum and Hygrophorus russula as noted in Table 1. 
WSRC-MS-2006-0422 Rev. 1

\section{Inocybes}

Most Inocybes are poisonous. They tend to have similar characteristics such as fibrous caps, gills and dark spore prints (spore prints of mushroom caps are often used for mushroom identification as noted in Læssøe (2002), McKnight and McKnight (1987) and Roody (2003). Depending on the species, they can be found in a variety of habitats and many will grow in bare soil, in the absence of decaying wood. Table 1 presents data for Inocybes which indicate that radiocesium activity concentrations are typically low but some may be as high as $\sim 900 \mathrm{~Bq}{ }^{137} \mathrm{Cs} / \mathrm{kg}$. No $\mathrm{T}_{\text {ag }}$ or TF values were reported as noted in Table 1.

\section{$\underline{\text { Laccaria and Lactarius }}$}

When young, the Laccaria amethystina are easily recognized because of its 2 to $5 \mathrm{~cm}$ diameter amethyst-colored cap and gills. However, with age this Laccaria species which grows in the forest litter of moist woodlands, can be confused with other members of its genus. Laccaria amethystina can accumulate large amounts of ${ }^{137} \mathrm{Cs}$ - up to nearly $120000 \mathrm{~Bq} / \mathrm{kg}$ (Table 1). The Lactarius mushrooms accumulate several tens of thousands of $\mathrm{Bq} / \mathrm{kg}$ of ${ }^{137} \mathrm{Cs}$ (Table 1). All members of the Lactarius genus uniquely exude a white or colored milk-like substance from their "crumbly" flesh when it is ruptured. Examples of these mushrooms are the poisonous Lactarius necator, L. rufus and the edible $L$. deliciosus.

For Lactarius, the $\mathrm{T}_{\text {ag }}$ and TF values were highly variable-ranging from $\mathrm{T}_{\text {ag }}$ values of 0.00007 to 0.068 and TF values of 0 to a high of 23.7. This suggests that the accumulation behavior of this genus varies widely. There is limited data for Laccaria but the reported TF values are high (0.36 to 3.0) relative to other species in Table 1.

\section{Lepiota (or Macrolepiota) procera, Lepista sp. and Mycena galericulata}

Lepiota (or Macrolepiota) procera or the parasol mushroom has an umbrella like shape and is edible. Their fruiting bodies of Lepista nuda and Mycena galericulata appear as troops and tufts on rotting stumps. These three fungi are common in temperate North America and Europe and they have been observed to accumulate a few hundred to a few thousand $\mathrm{Bq} / \mathrm{kg}$ of ${ }^{137} \mathrm{Cs}$ (Table 1).

As was the case for Lactarius, the $\mathrm{T}_{\mathrm{ag}}$ values for Lepiota procera, Lepista and Mycena galericulata were highly variable-ranging from $\mathrm{T}_{\text {ag }}$ values of 0.003 to 0.21 . However, the TF values were lower and ranged from 0.003 to 0.084 . These observations suggest that the accumulation behavior of this genus can vary.

\section{Paxillus involutus, Rhodophyllus and Russula sp.}

Paxillus involutus can accumulate nearly one million Bq/kg of ${ }^{137} \mathrm{Cs}$ (Table 1; Vinichuk and Johanson, 2003). This rather unprecedented amount of ${ }^{137} \mathrm{Cs}$ uptake was associated with mushrooms that were collected in the Ukraine following the Chernobyl accident. Paxillus involutus mushrooms are fairly common. They are found in conifer or birch woodlands and in gardens and parks. Fortunately, Paxillus involutus are poisonous and not consumed by humans. 
WSRC-MS-2006-0422 Rev. 1

The Russula mushrooms require symbiosis with trees or occasionally with shrubs in the form of mycorrhizal associations. Some Russula mushrooms are not edible whereas Rhodophyllus crassipes (or Entoloma rhodopolium) are poisonous. Russula and Rhodophyllus mushrooms tend to accumulate a few hundred to a few thousand $\mathrm{Bq} / \mathrm{kg}$ of ${ }^{137}$ Cs (Table 1).

TF values for Paxillus involutus mushrooms range form 2.01 to 21.6. These values are somewhat expected given the high levels of ${ }^{37} \mathrm{Cs}$ that were found to be present. When the levels of ${ }^{137} \mathrm{Cs}$ were ratioed to Boletus badius, in the study by Kammerer et al. (1994), the ratio was slightly lower than higherst value for Russula olchroleuca mushrooms, which had lower reported TF and $\mathrm{T}_{\mathrm{ag}}$ values than that of Paxillus involutus. These findings indicate some likely inconsistencies in the methods (TF, ratio values etc...) used to report these data from study to study.

Sarcodon imbricatus, Suillus sp., Tylopilus felleus and Tricholoma flavovirens

Sarcodon imbricatus are found in conifer as well as deciduous forests and it has been observed to accumulate close to $100000 \mathrm{~Bq} / \mathrm{kg}$ of ${ }^{137} \mathrm{Cs}$ (Table 1). They are edible although some have been poisoned by this mushroom. The Tylopilus felleus and Suillus mushrooms are also boletes. All of these mushrooms can accumulate a few thousand to a hundred thousand $\mathrm{Bq} / \mathrm{kg}$ of ${ }^{137} \mathrm{Cs}$ (Table 1). As was the case with TF data for Lactarius olchroleuca and Paxillus involutus, the TF values for Sarcodon imbricatus were high (22.2).

The Tricholomas or trichs typically grow on the ground rather than on a live or decaying wood form. They are perhaps best identified with the use of a field guide because they have a variety of colors, sizes and shapes, which can be confused with many other fungi. Tricholoma flavovirens, which accumulates moderate levels of radiocesium (from a few hundred to several thousand $\mathrm{Bq}{ }^{137} \mathrm{Cs} / \mathrm{kg}$ as listed in Table 1). No $\mathrm{T}_{\text {ag }}$ or TF values were reported for the Sarcodon imbricatus, Suillus and Tricholoma flavovirens mushrooms as noted in Table 1. Tylopilus felleus had TF values as high as 4.0, which based on comparison to mushrooms with similar ${ }^{137} \mathrm{Cs}$ concentrations (in Table 1), is somewhat to be expected.

\section{Mushrooms with a Low Affinity for Cs Accumulation}

Although not the focus of this review, several types of mushrooms often exhibit a low affinity for radiocesium. These observations are fairly independent of whether the mushrooms were sampled before or after the Chernobyl accident. Some Agaricus mushrooms, which are found in fields that contain livestock and in forests in addition to Coriolus hirstus and Pycnoporus coccineus do not exhibit a great propensity for Cs uptake (Byrne, 1988; Yoshida et al., 1994). Parasitic fungi such as the Armillaria mellea and Pholiota aegerita also exhibit low-to moderate uptake of radiocesium (less than a thousand $\mathrm{Bq} / \mathrm{kg}{ }^{137} \mathrm{Cs}$ ) relative to some of the fungi that were discussed previously (Battison et al., 1989; Yoshida et al., 1994; Toal et al., 2002). Studies with collections of Bulgaria inquinans, Calvatia excipuliformis, Lyophyllum sp., Lycoperdon perlatum, Asterophora lycoperdoides, Bulgaria inquinans and Calvatia excipuliformi have found that that these species typically do not have a large activity level of radiocesium relative to that of most of the species that are listed in Table 1 (Watling et al., 1993; Byrne, 1988;

Battison et al., 1989; Yoshida et al., 1994). For example mushrooms such as Lycoperdon perlatum typically accumulate $310 \mathrm{~Bq} / \mathrm{kg}$ of ${ }^{137} \mathrm{Cs}$ and $140 \mathrm{~Bq} / \mathrm{kg}$ of ${ }^{134} \mathrm{Cs}$ or less (Byrne, 
1988). In contrast, the mushroom Lycoperdon pyriforme had order of magnitude higher ${ }^{137} \mathrm{Cs}$ activity concentration than Lycoperdon perlatum but what is most noteworthy is its high $\mathrm{T}_{\text {ag }}$ factor (Toal et al. 2002) relative to other mushrooms that have much higher ${ }^{137} \mathrm{Cs}$ activity concentrations. Agrocybes typically accumulate low levels of ${ }^{137} \mathrm{Cs}$ but Agrocybe erebia can accumulate slightly more than $1500 \mathrm{~Bq} / \mathrm{kg}$ of ${ }^{137} \mathrm{Cs}$ or less (Table 1).

\section{Conclusions}

As previously discussed, some of the primary influences on ${ }^{134,137} \mathrm{Cs}$ uptake by mushrooms are growth media, the amount of deposition upon release, soil or growth media characteristics, growth habitat (saprophytic, parasitic or symbiotic), the species of mushroom (somewhat related to the habitat choice) and other yet to be discovered factors. The source term (such as whether the radiocesium is from Chernobyl or fallout from nuclear weapons testing) and other factors as mentioned have less affect on Cs accumulation. From these studies, it is clear that several mushrooms that demonstrate a high affinity for ${ }^{134,137} \mathrm{Cs}$ uptake are edible and are fairly common. Mushrooms are also eaten by some animals such as deer, which may be consumed by man. Regular consumption of these types of species or animals that eat these mushroom species may pose a human health concern.

\section{Acknowledgements}

This review was supported by the National Nuclear Security Administration's Office of Nonproliferation Research and Engineering (NA-22).

\section{Literature Cited}

Baeza, A., Guillén, J., and J. W. Mietelski. (2004). Uptake of alpha and beta emitters by mushrooms collected and cultured in Spain. J. Radioanal. Nucl. Chem. 261:375-380.

Battiston, G. A., Degetto, S., Gerbasi, R., and G. Sbrignadello. (1989). Radioactivity in mushrooms in Northeast Italy following the Chernobyl accident. J. Env. Radioact. 9:53-60.

Byrne, A. R. (1988). Radioactivity in fungi in Slovenia, Yugoslavia, following the Chernobyl accident. J. Env. Radioact. 6:177-183.

Cawes, P. A. and A. D. Horrill. (1986). A survey of caesium137 and plutonium in British soils in 1977. UKAEA, Harwell, p. 55.

Clint, G. M., Dighton, J., and S. Rees. (1991). Influx of ${ }^{137} \mathrm{Cs}$ into hyphae of basidiomycete fungi. Mycol. Res. 95:1047-1051.

Cooper, E. L., Zeller, E., Ghods-Esphahani, A., Makarewicz, M., Schlenz, R., Frindik, O., Heilgeist, M. and W. Kalus. (1992). Radioactivity in food and total diet samples collected in selected settlements in the USSR. J. Env. Radioact. 17:147-157.

Dighton, J. D. and A. D. Horrill. (1988). Radiocaesium accumulation in the mycorrhizal fungi Lactarius rufus and Inocybe longicystis in upland Britain following the Chernobyl accident. Trans. Brit. Mycol. Soc. 91:335-337. 
Grueter, H. (1964). Eine Selecktive Anreicherung des Spalkproduktes ${ }^{137} \mathrm{Cs}$ in Pilzen. Naturwissenschaten 51:161-162.

Grueter, H. (1971). Radioactive fission product ${ }^{137} \mathrm{Cs}$ in mushrooms in W. Germany during 1963-1970. Health Physics 20:655-656.

Guillitte, O., Gasia, M. C., Lambinon, J., Fraiture, A., Colard, J., and R. Kirchmann. (1987). La radiocontamination des champignons sauvages en Belgique et au Grand-Duche de Luxembourg apres II' accident nucleaire de tchernobyl. Mem. Soc. Roy. Botan. Belgium 9:79-93.

Haselwandter, K. (1978). Accumulation of the radioactive nuclide ${ }^{137} \mathrm{Cs}$ in fruitbodies of Basidomycetes. Health Physics 34:713-715.

Haselwandter, K, and M. Berreck (1994). Accumulation of radionuclides in fungi. In: Winkelmann, G. and D. R. Winge (eds) Metal ions in fungi. Marcel Dekker, New York.

Heinrich, G. (1993). Distribution of radiocesium in the different parts of mushrooms. J. Env. Radioact. 18:229-245.

Kammerer, L., Hiersche, L., and E. Wirth. (1994). Uptake of radiocaesium by different species of mushrooms. J. Env. Radioact. 23:135-150.

Læssøe, T. (2002). Mushrooms - Smithsonian Handbooks (G. Lincoff, Editorial Consultant), Dorling Kindersley Publishers, New York, NY.

McKnight, K. H. and V. B. McKnight. (1987). A field guide to mushrooms of North America. Peterson Field Guides. Houghton Mifflin Co. Publishers, New York, NY.

Muramatsu, Y., Yoshida, S., and M. Sumiya. (1991). Concentrations of radiocesium and potassium in basidiomycetes collected in Japan. Sci. Total Environ. 105:29-39.

Oolbekkink, G. T. and T. W. Kuyper. (1989). Radioactive caesium from Chernobyl in fungi. The Mycologist 3:3-6.

Řanda, Z. and J. Kučera. (2004). Trace elements in higher fungi (mushrooms) determined by activation analyses. J. Radioanal. Nucl. Chem. 259:99-107.

Roody, W. C. (2003). Mushrooms of West Virginia and the Central Appalachians. The University of Press of Kentucky, Lexington, KY.

Simkiss, K., Baxter, M. S., Bell, J. N. B., Davidson, W., Duncan, K., Fry, F., Horrill, A. D., Kelly, M., Mather, J. D., and J. W. Parsons. (1993). Radiocaesium in natural systems - A UK coordinated study. J. Env. Radioact. 18:133-149.

Toal, M. E., Copplestone, D., Johnson, M. S., Jackson, D., and S. R. Jones. (2002). Quantifying ${ }^{137} \mathrm{Cs}$ aggregated transfer coefficients in a semi-natural woodland ecosystem adjacent to a nuclear reprocessing facility. J. Env. Radioact. 63:85-103.

Vinichuk, M. M. and K. J. Johanson. (2003). Accumulation of ${ }^{137} \mathrm{Cs}$ by fungal mycelium in forest ecosystems of Ukraine. J. Env. Radioact. 64:27-43. 
Yoshida, S. and Y. Muramatsu. (1994). Accumulation of radiocesium in basidiomycetes collected from Japanese forests. Sci. Total Environ. 157:197-205.

Yoshida, S., Muramatsu, Y., and M. Ogawa. (1994). Radiocesium concentrations in mushrooms collected in Japan. J. Env. Radioact. 22:141-154.

Watling, R., Laessoe, T., Whalley, A. J. S., and N. W. Lepp. (1993). Radioactive caesium in British mushrooms. Botan. J. Scotland 46:487-497. 
Table 1. Radiocesium data for mushrooms that have a high affinity for Cs before and after Chernobyl. Concentration ratios (when reported) vary widely with mushroom radiocesium levels.

\begin{tabular}{|c|c|c|c|c|c|c|c|c|c|}
\hline $\begin{array}{l}\text { Cs } \\
\text { Iso- } \\
\text { tope }\end{array}$ & $\begin{array}{c}\text { Species Latin } \\
\text { Name }\end{array}$ & $\begin{array}{l}\text { Species } \\
\text { Common } \\
\text { Name }\end{array}$ & Country & $\begin{array}{l}\text { Location of } \\
\text { Study }\end{array}$ & Date & $\begin{array}{c}\text { Sampling Details } \\
\text { and Other } \\
\text { Observations }\end{array}$ & $\begin{array}{c}\text { Cs Activity } \\
\text { Level or } \\
\text { Ratioed } \\
\text { value* } \\
\end{array}$ & $\begin{array}{c}\text { Transfer } \\
\text { Factor or } \\
\mathbf{T}_{\mathrm{ag}}\end{array}$ & $\begin{array}{c}\text { Source of } \\
\text { Information }\end{array}$ \\
\hline 137 & $\begin{array}{l}\text { Agrocybe } \\
\text { erebia }\end{array}$ & $\begin{array}{l}\text { Leather } \\
\text { earthscale }\end{array}$ & Japan & Akita & $\begin{array}{l}1987, \\
1990\end{array}$ & $\begin{array}{l}\text { In fruiting body; } \\
\text { Edible; Saprotrophic }\end{array}$ & $1520 \mathrm{~Bq} / \mathrm{kg}$ & No data & $\begin{array}{l}\text { Muramatsu et al. } \\
\text { (1991); Yoshida and } \\
\text { Muramatsu (1994) }\end{array}$ \\
\hline 134 & $\begin{array}{l}\text { Agrocybe } \\
\text { erebia }\end{array}$ & $\begin{array}{l}\text { Leather } \\
\text { earthscale }\end{array}$ & Japan & Akita & $\begin{array}{l}1987, \\
1990\end{array}$ & $\begin{array}{l}\text { In fruiting body; } \\
\text { Edible; Saprotrophic }\end{array}$ & $97 \mathrm{~Bq} / \mathrm{kg}$ & No data & $\begin{array}{l}\text { Muramatsu et al. } \\
\text { (1991); Yoshida and } \\
\text { Muramatsu (1994) }\end{array}$ \\
\hline 137 & $\begin{array}{l}\text { Amanita } \\
\text { muscaria }\end{array}$ & $\begin{array}{c}\text { Fly amanita or } \\
\text { agaric }\end{array}$ & Spain & Muňoveros & $\begin{array}{l}1987 \\
1990\end{array}$ & $\begin{array}{l}\text { In fruiting body; } \\
\text { Poisonous }\end{array}$ & $5 \mathrm{~Bq} / \mathrm{kg}$ & No data & Baeza et al. (2004) \\
\hline 137 & $\begin{array}{l}\text { Amanita } \\
\text { muscaria }\end{array}$ & $\begin{array}{c}\text { Fly amanita or } \\
\text { agaric }\end{array}$ & Ukraine & $\begin{array}{l}\text { Zhitomir } \\
\text { region }\end{array}$ & $\begin{array}{l}1996- \\
1998\end{array}$ & $\begin{array}{l}\text { In fruiting body; } \\
\text { From Scots pine and } \\
\text { birch forests }\end{array}$ & $\begin{array}{c}8700 \text { to } \\
13400 \mathrm{~Bq} / \mathrm{kg}\end{array}$ & TF: 1.0 & $\begin{array}{l}\text { Vinichuk and } \\
\text { Johanson (2003) }\end{array}$ \\
\hline 137 & $\begin{array}{l}\text { Amanita } \\
\text { muscaria }\end{array}$ & $\begin{array}{c}\text { Fly amanita or } \\
\text { agaric }\end{array}$ & Austria & $\begin{array}{l}\text { State of } \\
\text { Styria }\end{array}$ & $\begin{array}{l}1987- \\
1989\end{array}$ & $\begin{array}{l}{ }^{137} \mathrm{Cs} \text { was mostly in } \\
\text { lamellae (gills) and } \\
\text { cap; From } 500 \text { and } \\
800 \mathrm{~m} \text { asl in spruce } \\
\text { and fir forests }\end{array}$ & $\begin{array}{l}1 \text { to } 250 \\
\mathrm{~Bq} / \mathrm{kg} \mathrm{fw}\end{array}$ & $\begin{array}{c}\mathrm{TF}: 0 \text { to } \\
0.25\end{array}$ & Heinrich (1993) \\
\hline 137 & $\begin{array}{c}\text { Amanita } \\
\text { ponderosa }\end{array}$ & & Spain & Aracena & $\begin{array}{l}1987 \\
1990\end{array}$ & In fruiting body & $5 \mathrm{~Bq} / \mathrm{kg}$ & No data & Baeza et al. (2004) \\
\hline 137 & $\begin{array}{l}\text { Amanita } \\
\text { porphyria }\end{array}$ & $\begin{array}{l}\text { Grey veiled or } \\
\text { Purple brown } \\
\text { amanita; } \\
\text { Porphyry } \\
\text { deathcap } \\
\end{array}$ & Germany & $\begin{array}{l}\text { Southern } \\
\text { Bavaria }\end{array}$ & $\begin{array}{l}1987- \\
1990\end{array}$ & $\begin{array}{l}\text { In fruiting body } \\
\text { (assumed) }\end{array}$ & $\begin{array}{l}\text { Ratioed to } B . \\
\text { badiu } \\
0.42 \text { to } 2.52\end{array}$ & No data & $\begin{array}{l}\text { Kammerer et al. } \\
(1994)\end{array}$ \\
\hline $\begin{array}{l}\text { Stable } \\
{ }^{133} \mathrm{Cs}\end{array}$ & $\begin{array}{l}\text { Amanita } \\
\text { rubescens }\end{array}$ & $\begin{array}{l}\text { The blusher; } \\
\text { Blushing } \\
\text { amanita }\end{array}$ & $\begin{array}{l}\text { Czech } \\
\text { Republic }\end{array}$ & Forests & $\begin{array}{c}\text { Late } \\
1990 \text { 's, } \\
\text { Early } \\
2000 \\
\end{array}$ & $\begin{array}{l}\text { In fruiting body; } \\
\text { Edible }\end{array}$ & $\begin{array}{l}\text { Up to } 1 \\
\mathrm{mg} / \mathrm{kg}\end{array}$ & No data & $\begin{array}{l}\text { Řanda and Kučera } \\
(2004)\end{array}$ \\
\hline 137 & $\begin{array}{l}\text { Amanita } \\
\text { rubescens }\end{array}$ & $\begin{array}{l}\text { The blusher; } \\
\text { Blushing } \\
\text { amanita }\end{array}$ & Austria & $\begin{array}{l}\text { State of } \\
\text { Styria }\end{array}$ & $\begin{array}{c}1987- \\
1989\end{array}$ & $\begin{array}{l}{ }^{137} \text { Cs was mostly in } \\
\text { lamellae and cap; } \\
\text { From } 500 \text { to } 1000 \mathrm{~m} \\
\text { asl spruce and fir } \\
\text { forests }\end{array}$ & $\begin{array}{c}2001 \text { to } 4000 \\
\mathrm{~Bq} / \mathrm{kg} \mathrm{fw}\end{array}$ & $\begin{array}{c}\mathrm{TF}: 0 \text { to } \\
0.25\end{array}$ & Heinrich (1993) \\
\hline 137 & $\begin{array}{l}\text { Amanita } \\
\text { rubescens }\end{array}$ & $\begin{array}{c}\text { The blusher; } \\
\text { Blushing } \\
\text { amanita }\end{array}$ & $\begin{array}{l}\text { United } \\
\text { Kingdom }\end{array}$ & $\begin{array}{l}\text { Lady Wood } \\
\text { Forest, } 0.5 \\
\text { km from } \\
\text { Sellafield }\end{array}$ & $\begin{array}{l}\text { Summe } \\
\text { r } 1996\end{array}$ & In fruiting body & $\begin{array}{l}150 \text { to } 220 \\
\mathrm{~Bq} / \mathrm{kg}\end{array}$ & $\begin{array}{l}\mathrm{T}_{\mathrm{ag}}: 0.0058 \\
\text { to } 0.0085\end{array}$ & Toal et al. (2002) \\
\hline 137 & $\begin{array}{l}\text { Amanita } \\
\text { rubescens }\end{array}$ & $\begin{array}{c}\text { The blusher; } \\
\text { Blushing } \\
\text { amanita }\end{array}$ & $\begin{array}{l}\text { United } \\
\text { Kingdom }\end{array}$ & Prescot & $\begin{array}{l}1987 \\
1990\end{array}$ & $\begin{array}{l}\text { In fruiting body; } \\
\text { Ectomycorrhizal } \\
\text { species }\end{array}$ & $45.5 \mathrm{~Bq} / \mathrm{kg}$ & No data & Watling et al. (1993) \\
\hline 137 & $\begin{array}{l}\text { Boletus (or } \\
\text { Xerocomus) } \\
\text { subtomenosus }\end{array}$ & $\begin{array}{l}\text { Yellow } \\
\text { cracked } \\
\text { boletus }\end{array}$ & Austria & $\begin{array}{l}\text { State of } \\
\text { Styria }\end{array}$ & $\begin{array}{c}1987- \\
1989\end{array}$ & $\begin{array}{l}{ }^{137} \text { Cs was mostly in } \\
\text { lamellae; From } 500 \\
\text { and } 800 \mathrm{~m} \text { asl in } \\
\text { spruce and fir forests }\end{array}$ & $\begin{array}{l}501 \text { to } 1000 \\
\mathrm{~Bq} / \mathrm{kg} \mathrm{fw}\end{array}$ & $\begin{array}{c}\mathrm{TF}: 0 \text { to } \\
0.25\end{array}$ & Heinrich (1993) \\
\hline 137 & $\begin{array}{l}\text { Boletus (or X.) } \\
\text { subtomenosus }\end{array}$ & $\begin{array}{l}\text { Yellow } \\
\text { cracked } \\
\text { boletus }\end{array}$ & Ukraine & $\begin{array}{l}\text { Zhitomir } \\
\text { Region }\end{array}$ & $\begin{array}{c}1996- \\
1998\end{array}$ & $\begin{array}{l}\text { In fruiting body; } \\
\text { From Scots pine and } \\
\text { birch forests }\end{array}$ & $\begin{array}{c}20500 \text { to } \\
117200 \\
\mathrm{~Bq} / \mathrm{kg} \\
\end{array}$ & $\mathrm{TF}: 3.1$ & $\begin{array}{l}\text { Vinichuk and } \\
\text { Johanson (2003) }\end{array}$ \\
\hline 137 & $\begin{array}{l}\text { Boletus (or X.) } \\
\text { subtomentosus }\end{array}$ & $\begin{array}{l}\text { Yellow } \\
\text { cracked } \\
\text { boletus }\end{array}$ & $\begin{array}{l}\text { United } \\
\text { Kingdom }\end{array}$ & $\begin{array}{l}\text { Swallow } \\
\text { Falls }\end{array}$ & $\begin{array}{c}1987 \\
1990\end{array}$ & $\begin{array}{l}\text { In fruiting body; } \\
\text { Ectomycorrhizal } \\
\text { species }\end{array}$ & $\begin{array}{c}\text { Below } \\
\text { detection }\end{array}$ & No data & Watling et al. (1993) \\
\hline 137 & $\begin{array}{l}\text { Boletus (or } \\
\text { Xerocomus) } \\
\quad \text { badius }\end{array}$ & Bay bolete & Germany & $\begin{array}{l}\text { Western } \\
\text { Germany }\end{array}$ & 1966 & $\begin{array}{l}\text { In fruiting body } \\
\text { (assumed); Soil } \\
\text { cleaned off prior to } \\
\text { analysis }\end{array}$ & $\begin{array}{c}1133 \mathrm{~Bq} / \mathrm{kg} \\
\mathrm{fw}\end{array}$ & No data & Greuter (1971) \\
\hline
\end{tabular}


WSRC-MS-2006-0422 Rev. 1

\begin{tabular}{|c|c|c|c|c|c|c|c|c|c|}
\hline 137 & $\begin{array}{l}\text { Boletus (or X) } \\
\text { badius }\end{array}$ & Bay bolete & Austria & $\begin{array}{l}\text { State of } \\
\text { Styria }\end{array}$ & $\begin{array}{l}1987- \\
1989\end{array}$ & $\begin{array}{l}{ }^{137} \text { Cs was mostly in } \\
\text { lamellae and cap; } \\
\text { From } 500 \text { and } 800 \mathrm{~m} \\
\text { asl in spruce and fir } \\
\text { forests }\end{array}$ & $\begin{array}{c}8001 \text { to } \\
16000 \mathrm{~Bq} / \mathrm{kg} \\
\text { fw }\end{array}$ & $\begin{array}{c}\mathrm{TF}: 1.01 \text { to } \\
2.0\end{array}$ & Heinrich (1993) \\
\hline 137 & $\begin{array}{l}\text { Boletus (or } X) \\
\text { badius }\end{array}$ & Bay bolete & Germany & $\begin{array}{l}\text { Southern } \\
\text { Bavaria }\end{array}$ & $\begin{array}{l}1987- \\
1990\end{array}$ & $\begin{array}{l}\text { In fruiting body } \\
\text { (assumed) }\end{array}$ & $\begin{array}{c}1320 \text { to } 4365 \\
\mathrm{~Bq} / \mathrm{kg} \mathrm{fw}\end{array}$ & $\begin{array}{l}\text { TF: } 0.7 \text { to } \\
5.0 ; \\
\text { Mean: } 2.0\end{array}$ & $\begin{array}{l}\text { Kammerer et al. } \\
\text { (1994) }\end{array}$ \\
\hline $\begin{array}{l}134 \\
137\end{array}$ & $\begin{array}{c}\text { Boletus (or } X . \text { ) } \\
\text { badius }\end{array}$ & Bay bolete & $\begin{array}{l}\text { Yugoslav } \\
\text {-ia }\end{array}$ & Slovenia & 1986 & All; After Chernobyl & $\begin{array}{c}1200 \text { to } \\
66000 \mathrm{~Bq} \\
{ }^{134,137} \mathrm{Cs} / \mathrm{kg} \\
\text { Mean: } 19000 \\
\mathrm{~Bq} \\
{ }^{134,137} \mathrm{Cs} / \mathrm{kg}\end{array}$ & No data & Byrne (1988) \\
\hline 137 & $\begin{array}{l}\text { Boletus } \\
\text { calopus }\end{array}$ & $\begin{array}{l}\text { Scarlet- } \\
\text { stemmed } \\
\text { bolete }\end{array}$ & $\begin{array}{l}\text { United } \\
\text { Kingdom }\end{array}$ & $\begin{array}{l}\text { Lady Wood } \\
\text { Forest, } 0.5 \\
\text { km from } \\
\text { Sellafield }\end{array}$ & $\begin{array}{c}\text { Autumn } \\
1996\end{array}$ & $\begin{array}{l}\text { In fruiting body; } \\
\text { Cleaned before } \\
\text { analyses; From a } \\
\text { Picea sitchensis } \\
\text { woodland }\end{array}$ & $71.0 \mathrm{~Bq} / \mathrm{kg}$ & $\mathrm{T}_{\mathrm{ag}}: 0.0027$ & Toal et al. (2002) \\
\hline 137 & $\begin{array}{l}\text { Boletus (or } \\
\text { Xerocomus) } \\
\text { chrysenteron }\end{array}$ & $\begin{array}{l}\text { Red cracking } \\
\text { bolete }\end{array}$ & $\begin{array}{l}\text { United } \\
\text { Kingdom }\end{array}$ & $\begin{array}{l}\text { Lady Wood } \\
\text { Forest, } 0.5 \\
\text { km from } \\
\text { Sellafield }\end{array}$ & $\begin{array}{c}\text { Autumn } \\
1996\end{array}$ & $\begin{array}{l}\text { In fruiting body; } \\
\text { Cleaned before } \\
\text { analyses; From a } \\
\text { Picea sitchensis } \\
\text { woodland }\end{array}$ & $\begin{array}{c}1345 \text { to } 1570 \\
\mathrm{~Bq} / \mathrm{kg}\end{array}$ & $\mathrm{T}_{\mathrm{ag}}: 0.056$ & Toal et al. (2002) \\
\hline 137 & $\begin{array}{c}\text { Boletus (or X.) } \\
\text { chrysenteron }\end{array}$ & $\begin{array}{l}\text { Red cracking } \\
\text { bolete }\end{array}$ & Austria & $\begin{array}{l}\text { State of } \\
\text { Styria }\end{array}$ & $\begin{array}{l}1987- \\
1989\end{array}$ & $\begin{array}{l}{ }^{137} \text { Cs was mostly in } \\
\text { lamellae and cap; } \\
\text { From } 500 \text { and } 800 \mathrm{~m} \\
\text { asl in spruce and fir } \\
\text { forests }\end{array}$ & $\begin{array}{c}8001 \text { to } \\
16000 \mathrm{~Bq} / \mathrm{kg} \\
\text { fw }\end{array}$ & $\mathrm{TF}:>4.0$ & Heinrich (1993) \\
\hline 137 & Boletus edulis & $\begin{array}{l}\text { King or edible } \\
\text { bolete, cep, } \\
\text { cepe, porcini } \\
\text { or Penny-bun }\end{array}$ & Ukraine & $\begin{array}{l}\text { Zhitomir } \\
\text { Region }\end{array}$ & $\begin{array}{l}1996- \\
1998\end{array}$ & $\begin{array}{l}\text { In fruiting body; } \\
\text { From Scots pine and } \\
\text { birch forests }\end{array}$ & $\begin{array}{c}2000 \text { to } \\
41200 \mathrm{~Bq} / \mathrm{kg}\end{array}$ & TF:2.3 & $\begin{array}{l}\text { Vinichuk and } \\
\text { Johanson (2003) }\end{array}$ \\
\hline 137 & Boletus edulis & $\begin{array}{l}\text { King or edible } \\
\text { bolete, cep, } \\
\text { cepe, porcini } \\
\text { or Penny-bun }\end{array}$ & Austria & $\begin{array}{l}\text { State of } \\
\text { Styria }\end{array}$ & $\begin{array}{c}1987- \\
1989\end{array}$ & $\begin{array}{l}{ }^{137} \text { Cs was mostly in } \\
\text { lamellae and cap; } \\
\text { From } 500 \text { and } 800 \mathrm{~m} \\
\text { asl in spruce and fir } \\
\text { forests }\end{array}$ & $\begin{array}{l}501 \text { to } 1000 \\
\mathrm{~Bq} / \mathrm{kg} \mathrm{fw}\end{array}$ & $\begin{array}{c}\mathrm{TF}: 0 \text { to } \\
0.25\end{array}$ & Heinrich (1993) \\
\hline $\begin{array}{l}\text { Stable } \\
{ }^{133} \mathrm{Cs}\end{array}$ & Boletus edulis & $\begin{array}{l}\text { King or edible } \\
\text { bolete, cep, } \\
\text { cepe, porcini } \\
\text { or Penny-bun }\end{array}$ & $\begin{array}{c}\text { Czech } \\
\text { Republic }\end{array}$ & Forests & $\begin{array}{l}\text { Late } \\
1990 \text { 's, } \\
\text { Early } \\
2000\end{array}$ & In fruiting body & $\begin{array}{l}\text { Up to } 2.73 \\
\mathrm{mg} / \mathrm{kg}\end{array}$ & No data & $\begin{array}{l}\text { Řanda and Kučera } \\
\text { (2004) }\end{array}$ \\
\hline 137 & Boletus edulis & $\begin{array}{l}\text { King or edible } \\
\text { bolete, cep, } \\
\text { cepe, porcini } \\
\text { or Penny-bun }\end{array}$ & $\begin{array}{l}\text { United } \\
\text { Kingdom }\end{array}$ & Linn Park & $\begin{array}{l}1987, \\
1990\end{array}$ & $\begin{array}{l}\text { In fruiting body; } \\
\text { Ectomycorrhizal } \\
\text { species }\end{array}$ & $68.4 \mathrm{~Bq} / \mathrm{kg}$ & No data & Watling et al. (1993) \\
\hline $\begin{array}{l}\text { Stable } \\
{ }^{133} \mathrm{Cs}\end{array}$ & $\begin{array}{l}\text { Boletus (or } \\
\text { Suillus) } \\
\text { variegatus }\end{array}$ & $\begin{array}{l}\text { Variegated } \\
\text { bolete; Yel- } \\
\text { lowy-brown } \\
\text { mossiness } \\
\text { mushroom }\end{array}$ & $\begin{array}{c}\text { Czech } \\
\text { Republic }\end{array}$ & Forests & $\begin{array}{l}\text { Late } \\
1990 \text { 's, } \\
\text { Early } \\
2000\end{array}$ & In fruiting body & $\begin{array}{l}\text { Up to } 2.06 \\
\mathrm{mg} / \mathrm{kg}\end{array}$ & No data & $\begin{array}{l}\text { Řanda and Kučera } \\
\text { (2004) }\end{array}$ \\
\hline 137 & $\begin{array}{c}\text { Boletus (or S.) } \\
\text { variegatus }\end{array}$ & $\begin{array}{l}\text { Variegated } \\
\text { bolete; Yel- } \\
\text { lowy-brown } \\
\text { mossiness } \\
\text { mushroom }\end{array}$ & Germany & $\begin{array}{l}\text { Southern } \\
\text { Bavaria }\end{array}$ & $\begin{array}{c}1987- \\
1990\end{array}$ & $\begin{array}{l}\text { In fruiting body } \\
\text { (assumed) }\end{array}$ & $\begin{array}{l}\text { Ratioed to } B \text {. } \\
\text { badius**:0.3 } \\
7 \text { to } 0.55 \\
665 \text { to } 1250 \\
\mathrm{~Bq} / \mathrm{kg} \mathrm{fw}\end{array}$ & $\begin{array}{c}\mathrm{TF}: 0.98 \text { to } \\
1.1 ; \\
\text { Mean: } 1.0\end{array}$ & $\begin{array}{l}\text { Kammerer et al. } \\
\text { (1994) }\end{array}$ \\
\hline
\end{tabular}


WSRC-MS-2006-0422 Rev. 1

\begin{tabular}{|c|c|c|c|c|c|c|c|c|c|}
\hline 137 & $\begin{array}{c}\text { Boletus (or S.) } \\
\text { variegatus }\end{array}$ & $\begin{array}{l}\text { Variegated } \\
\text { bolete; Yel- } \\
\text { lowy brown } \\
\text { mossiness } \\
\text { mushroom }\end{array}$ & Ukraine & $\begin{array}{l}\text { Zhitomir } \\
\text { Region }\end{array}$ & $\begin{array}{l}1996- \\
1998\end{array}$ & $\begin{array}{l}\text { In fruiting body; } \\
\text { From Scots pine and } \\
\text { birch forests }\end{array}$ & $\begin{array}{c}\text { Mean:98800 } \\
\mathrm{Bq} / \mathrm{kg}\end{array}$ & $\mathrm{TF}: 2.6$ & $\begin{array}{l}\text { Vinichuk and } \\
\text { Johanson (2003) }\end{array}$ \\
\hline 137 & $\begin{array}{c}\text { Boletus (or S.) } \\
\text { variegatus }\end{array}$ & $\begin{array}{l}\text { Variegated } \\
\text { bolete; Yel- } \\
\text { lowy brown } \\
\text { mossiness } \\
\text { mushroom }\end{array}$ & Austria & $\begin{array}{l}\text { State of } \\
\text { Styria }\end{array}$ & $\begin{array}{l}1987- \\
1989\end{array}$ & $\begin{array}{l}{ }^{137} \text { Cs was mostly in } \\
\text { lamellae and cap; } \\
\text { From } 500 \text { and } 800 \mathrm{~m} \\
\text { asl in spruce and fir } \\
\text { forests }\end{array}$ & $\begin{array}{c}1001 \text { to } 2000 \\
\mathrm{~Bq} / \mathrm{kg} \mathrm{fw}\end{array}$ & $\begin{array}{c}\mathrm{TF}: 0.51 \text { to } \\
1.0\end{array}$ & Heinrich (1993) \\
\hline 137 & $\begin{array}{l}\text { Bulgaria } \\
\text { inquinans }\end{array}$ & $\begin{array}{l}\text { Black jelly- } \\
\text { drop cups }\end{array}$ & $\begin{array}{l}\text { United } \\
\text { Kingdom }\end{array}$ & Dollar & $\begin{array}{l}1987, \\
1990\end{array}$ & $\begin{array}{l}\text { In fruiting body; } \\
\text { Saprotrophic; } \\
\text { Growing on rotting } \\
\text { wood (lignin) }\end{array}$ & $390 \mathrm{~Bq} / \mathrm{kg}$ & No data & Watling et al. (1993) \\
\hline 137 & $\begin{array}{c}\text { Calvatia } \\
\text { excipuliformis }\end{array}$ & $\begin{array}{l}\text { Pestle-shaped } \\
\text { puffball }\end{array}$ & $\begin{array}{l}\text { United } \\
\text { Kingdom }\end{array}$ & Prescot & $\begin{array}{l}1987, \\
1990\end{array}$ & \begin{tabular}{|c|} 
In fruiting body; \\
Saprotrophic; \\
Growing on decaying \\
litter \\
\end{tabular} & $271.6 \mathrm{~Bq} / \mathrm{kg}$ & No data & Watling et al. (1993) \\
\hline 137 & $\begin{array}{l}\text { Cantharellus } \\
\text { cibarius }\end{array}$ & Chanterelle & Austria & $\begin{array}{l}\text { State of } \\
\text { Styria }\end{array}$ & $\begin{array}{c}1987- \\
1989\end{array}$ & $\begin{array}{l}{ }^{137} \text { Cs was mostly in } \\
\text { lamellae and cap; } \\
\text { From spruce and fir } \\
\text { forests at } 500 \mathrm{~m} \text { asl }\end{array}$ & $\begin{array}{c}1001 \text { to } 2000 \\
\mathrm{~Bq} / \mathrm{kg} \mathrm{fw}\end{array}$ & $\begin{array}{l}\mathrm{TF}: 1.01 \text { to } \\
2.0\end{array}$ & Heinrich (1993) \\
\hline 137 & $\begin{array}{l}\text { Cantharellus } \\
\text { cibarius }\end{array}$ & Chanterelle & Ukraine & $\begin{array}{l}\text { Zhitomir } \\
\text { Region }\end{array}$ & $\begin{array}{c}1996- \\
1998\end{array}$ & $\begin{array}{l}\text { In fruiting body; } \\
\text { From Scots pine and } \\
\text { birch forests }\end{array}$ & $\begin{array}{c}\text { Mean: } 15400 \\
\mathrm{~Bq} / \mathrm{kg}\end{array}$ & TF:5.0 & $\begin{array}{l}\text { Vinichuk and } \\
\text { Johanson (2003) }\end{array}$ \\
\hline $\begin{array}{l}\text { Stable } \\
{ }^{133} \mathrm{Cs}\end{array}$ & $\begin{array}{l}\text { Cantharellus } \\
\text { cibarius }\end{array}$ & Chanterelle & $\begin{array}{l}\text { Czech } \\
\text { Republic }\end{array}$ & Forests & $\begin{array}{c}\text { Late } \\
1990 \text { 's, } \\
\text { Early } \\
2000 \\
\end{array}$ & In fruiting body & $\begin{array}{c}\text { Mean:1.04 } \\
\mathrm{mg} / \mathrm{kg}\end{array}$ & No data & $\begin{array}{l}\text { Řanda and Kučera } \\
\text { (2004) }\end{array}$ \\
\hline 137 & $\begin{array}{l}\text { Cantharellus } \\
\text { cibarius }\end{array}$ & Chanterelle & $\begin{array}{l}\text { United } \\
\text { Kingdom }\end{array}$ & Cleish & $\begin{array}{c}1987 \\
1990\end{array}$ & $\begin{array}{l}\text { In fruiting body; } \\
\text { Ectomycorrhizal } \\
\text { species }\end{array}$ & $133 \mathrm{~Bq} / \mathrm{kg}$ & No data & Watling et al. (1993) \\
\hline 137 & $\begin{array}{l}\text { Cantharellus } \\
\text { lutescens }\end{array}$ & $\begin{array}{c}\text { Yellow(ish) or } \\
\text { golden } \\
\text { chanterelle }\end{array}$ & Italy & $\begin{array}{l}\text { Northeastern } \\
\text { Portion }\end{array}$ & 1986 & $\begin{array}{l}\text { All; Sampled after } \\
\text { Chernobyl }\end{array}$ & $\begin{array}{c}4991 \text { to } \\
27626 \mathrm{~Bq} / \mathrm{kg}\end{array}$ & $\begin{array}{c}\text { TF: } 0.125 \\
\text { to } 0.691\end{array}$ & $\begin{array}{l}\text { Battiston et al. } \\
\text { (1989) }\end{array}$ \\
\hline 137 & $\begin{array}{l}\text { Cantharellus } \\
\text { lutescens }\end{array}$ & $\begin{array}{l}\text { Yellow(ish) or } \\
\text { golden } \\
\text { chanterelle }\end{array}$ & Italy & $\begin{array}{l}\text { Northeastern } \\
\text { Portion }\end{array}$ & 1986 & $\begin{array}{l}\text { All; Does not include } \\
{ }^{137} \text { Cs from } \\
\text { Chernobyl }\end{array}$ & $\begin{array}{c}1071 \text { to } 3303 \\
\mathrm{~Bq} / \mathrm{kg}\end{array}$ & No data & $\begin{array}{l}\text { Battiston et al. } \\
\text { (1989) }\end{array}$ \\
\hline 134 & $\begin{array}{l}\text { Cantharellus } \\
\text { lutescens }\end{array}$ & $\begin{array}{l}\text { Yellow(ish) or } \\
\text { golden } \\
\text { chanterelle }\end{array}$ & Italy & $\begin{array}{l}\text { Northeastern } \\
\text { Portion }\end{array}$ & 1986 & $\begin{array}{c}\text { All; Does not include } \\
{ }^{137} \text { Cs from } \\
\text { Chernobyl }\end{array}$ & $\begin{array}{c}1894 \text { to } 4298 \\
\mathrm{~Bq} / \mathrm{kg}\end{array}$ & $\begin{array}{l}\text { TF:0.095 } \\
\text { to } 0.215\end{array}$ & $\begin{array}{l}\text { Battiston et al. } \\
\text { (1989) }\end{array}$ \\
\hline $\begin{array}{l}\text { Stable } \\
{ }^{133} \mathrm{Cs}\end{array}$ & $\begin{array}{l}\text { Cantharellus } \\
\text { lutescens }\end{array}$ & $\begin{array}{l}\text { Yellow(ish) or } \\
\text { golden } \\
\text { chanterelle }\end{array}$ & $\begin{array}{l}\text { Czech } \\
\text { Republic }\end{array}$ & Forests & $\begin{array}{l}\text { Late } \\
1990 \text { 's, } \\
\text { Early } \\
2000\end{array}$ & In fruiting body & $\begin{array}{c}\text { Mean: } 1.53 \\
\mathrm{mg} / \mathrm{kg}\end{array}$ & No data & $\begin{array}{l}\text { Řanda and Kučera } \\
\text { (2004) }\end{array}$ \\
\hline 137 & $\begin{array}{l}\text { Cantharellus } \\
\text { tubaeformis }\end{array}$ & $\begin{array}{c}\text { Funnel or } \\
\text { Trumpet } \\
\text { chanterelle }\end{array}$ & Germany & $\begin{array}{l}\text { Southern } \\
\text { Bavaria }\end{array}$ & $\begin{array}{c}1987- \\
1990\end{array}$ & $\begin{array}{l}\text { In fruiting body } \\
\text { (assumed) }\end{array}$ & \begin{tabular}{|c|} 
Ratioed to $B$. \\
badius**:0.3 \\
9 to $1.71 ;$ \\
1700 to 3270 \\
$\mathrm{~Bq} / \mathrm{kg} \mathrm{fw}$ \\
\end{tabular} & $\begin{array}{c}\text { TF: } 0.52 \text { to } \\
4.3 ; \\
\text { Mean: } 2.3\end{array}$ & $\begin{array}{l}\text { Kammerer et al. } \\
(1994)\end{array}$ \\
\hline 137 & $\begin{array}{l}\text { Clitocybe } \\
\text { infundibulifor- } \\
\text { mis orC. gibba }\end{array}$ & $\begin{array}{l}\text { Funnel cap or } \\
\text { clithocybe }\end{array}$ & Italy & & $\begin{array}{c}\text { August } \\
1986\end{array}$ & $\begin{array}{l}\text { All; Edible, After } \\
\text { Chernobyl }\end{array}$ & $\begin{array}{c}12030 \text { to } \\
24532 \mathrm{~Bq} / \mathrm{kg}\end{array}$ & $\begin{array}{l}\text { TF: } 0.523 \\
\text { to } 0.6133\end{array}$ & $\begin{array}{l}\text { Battiston et al. } \\
\text { (1989) }\end{array}$ \\
\hline 137 & $\begin{array}{l}\text { Clitocybe } \\
\text { infundibulifor- } \\
\text { mis orC. gibba }\end{array}$ & $\begin{array}{l}\text { Funnel cap or } \\
\text { clithocybe }\end{array}$ & Italy & & $\begin{array}{c}\text { August } \\
1986\end{array}$ & $\begin{array}{c}\text { All; Does not include } \\
{ }^{137} \text { Cs from } \\
\text { Chernobyl }\end{array}$ & $\begin{array}{c}1427 \text { to } 3303 \\
\mathrm{~Bq} / \mathrm{kg}\end{array}$ & No data & $\begin{array}{l}\text { Battiston et al. } \\
\text { (1989) }\end{array}$ \\
\hline 134 & $\begin{array}{l}\text { Clitocybe } \\
\text { infundibulifor- } \\
\text { mis orC. gibba }\end{array}$ & $\begin{array}{l}\text { Funnel cap or } \\
\text { clithocybe }\end{array}$ & Italy & & $\begin{array}{c}\text { August } \\
1986\end{array}$ & $\begin{array}{c}\text { All; Does not include } \\
{ }^{137} \text { Cs from } \\
\text { Chernobyl }\end{array}$ & $\begin{array}{c}5581 \text { to } \\
11331 \mathrm{~Bq} / \mathrm{kg}\end{array}$ & $\begin{array}{l}\text { TF: } 0.465 \\
\text { to } 0.5665\end{array}$ & $\begin{array}{l}\text { Battiston et al. } \\
\text { (1989) }\end{array}$ \\
\hline
\end{tabular}


WSRC-MS-2006-0422 Rev. 1

\begin{tabular}{|c|c|c|c|c|c|c|c|c|c|}
\hline 137 & $\begin{array}{l}\text { Cortinarius } \\
\text { armillatus }\end{array}$ & $\begin{array}{c}\text { Cinnabar } \\
\text { bracelet } \\
\text { webcap; Red } \\
\text { banded web- } \\
\text { cap or cort }\end{array}$ & Europe & $\begin{array}{c}\text { Sweden, } \\
\text { Switzerland, } \\
\text { Italy, Austria } \\
\text { and Finland }\end{array}$ & 1974 & In fruiting body & $\begin{array}{c}\text { Mean:5185 } \\
\mathrm{Bq} / \mathrm{kg}\end{array}$ & No data & Haselwandter (1978) \\
\hline $\begin{array}{l}134 \\
137\end{array}$ & $\begin{array}{l}\text { Cortinarius } \\
\text { armillatus }\end{array}$ & $\begin{array}{c}\text { Cinnabar } \\
\text { bracelet } \\
\text { webcap; Red } \\
\text { banded web- } \\
\text { cap or cort }\end{array}$ & $\begin{array}{l}\text { Yugo- } \\
\text { slavia }\end{array}$ & Slovenia & 1986 & $\begin{array}{l}\text { All portions; After } \\
\text { Chernobyl }\end{array}$ & $\begin{array}{c}21000 \text { to } \\
96000 \\
\mathrm{~Bq}{ }^{134,137} \mathrm{Cs} / \\
\mathrm{kg} ; \text { Mean: } \\
51000 \mathrm{~Bq} \\
{ }_{134,137} \mathrm{Cs} / \mathrm{kg}\end{array}$ & No data & Byrne (1988) \\
\hline 137 & $\begin{array}{l}\text { Cortinarius } \\
\text { caperatus or } \\
\text { Rozites } \\
\text { caperata }\end{array}$ & $\begin{array}{l}\text { The gypsy; } \\
\text { Gypsy nitecap } \\
\text { or rozites }\end{array}$ & Germany & $\begin{array}{l}\text { Southern } \\
\text { Bavaria }\end{array}$ & $\begin{array}{l}1987- \\
1990\end{array}$ & $\begin{array}{l}\text { All portions } \\
\text { (assumed) }\end{array}$ & $\begin{array}{l}\text { Ratioed to } B \text {. } \\
\text { badius } * * \text { : } \\
0.64 \text { to } 1.36 ; \\
2090 \text { to } 3070 \\
\text { Bq } / \mathrm{kg} \mathrm{fw}\end{array}$ & $\begin{array}{c}\text { TF:2.1 to } \\
3.3 ; \\
\text { Mean: } 2.8\end{array}$ & $\begin{array}{l}\text { Kammerer et al. } \\
(1994)\end{array}$ \\
\hline $\begin{array}{l}134 \\
137\end{array}$ & $\begin{array}{l}\text { Cortinarius } \\
\text { caperatus or } \\
\text { Rozites } \\
\text { caperata }\end{array}$ & $\begin{array}{l}\text { The gypsy; } \\
\text { Gypsy nitecap } \\
\text { or rozites }\end{array}$ & $\begin{array}{l}\text { Yugo- } \\
\text { slavia }\end{array}$ & Slovenia & 1986 & $\begin{array}{l}\text { All portions; Does } \\
\text { not include } \\
\text { Chernobyl fallout }\end{array}$ & $\begin{array}{c}2100 \text { to } \\
62000 \mathrm{~Bq} \\
{ }_{134,137} \mathrm{Cs} / \\
\mathrm{kg} ; \mathrm{Mean}: \\
22600 \mathrm{~Bq} \\
{ }_{134,137} \mathrm{Cs} / \mathrm{kg}\end{array}$ & No data & Byrne (1988) \\
\hline 137 & $\begin{array}{l}\text { Cortinarius } \\
\text { caperatus or } \\
\text { Rozites } \\
\text { caperata }\end{array}$ & $\begin{array}{l}\text { The gypsy; } \\
\text { Gypsy nitecap } \\
\text { or rozites }\end{array}$ & Austria & $\begin{array}{l}\text { State of } \\
\text { Styria }\end{array}$ & $\begin{array}{l}1987- \\
1989\end{array}$ & $\begin{array}{l}{ }^{137} \text { Cs was mostly in } \\
\text { cap and lamellae; } \\
\text { Found in a spruce } \\
\text { and fir forest soil; } \\
800 \text { and } 1000 \mathrm{~m} \text { asl }\end{array}$ & $\begin{array}{c}>32000 \\
\mathrm{~Bq} / \mathrm{kg} \mathrm{fw}\end{array}$ & TF: $>4.0$ & Heinrich (1993) \\
\hline 137 & $\begin{array}{l}\text { Cortinarius } \\
\text { caperatus or } \\
\text { Rozites } \\
\text { caperata }\end{array}$ & $\begin{array}{l}\text { The gypsy; } \\
\text { Gypsy nitecap } \\
\text { or rozites }\end{array}$ & Europe & $\begin{array}{c}\text { Sweden, } \\
\text { Switzerland, } \\
\text { Italy, Austria } \\
\text { and Finland }\end{array}$ & 1974 & In fruiting body & $\begin{array}{c}\text { Mean: } 10370 \\
\mathrm{~Bq} / \mathrm{kg}\end{array}$ & No data & Haselwandter (1978) \\
\hline $\begin{array}{l}\text { Stable } \\
{ }^{133} \mathrm{Cs}\end{array}$ & $\begin{array}{l}\text { Cortinarius } \\
\text { caperatus or } \\
\text { Rozites } \\
\text { caperata }\end{array}$ & $\begin{array}{l}\text { The gypsy; } \\
\text { Gypsy nitecap } \\
\text { or rozites }\end{array}$ & $\begin{array}{c}\text { Czech } \\
\text { Republic }\end{array}$ & Forests & $\begin{array}{l}\text { Late } \\
1990 \text { 's, } \\
\text { Early } \\
2000\end{array}$ & Fruiting body & $\begin{array}{c}\text { Mean:8.39 } \\
\mathrm{mg} / \mathrm{kg}\end{array}$ & No data & $\begin{array}{l}\text { Řanda and Kučera } \\
(2004)\end{array}$ \\
\hline 137 & $\begin{array}{l}\text { Cortinarius } \\
\text { intergerrimus }\end{array}$ & & Austria & $\begin{array}{l}\text { State of } \\
\text { Styria }\end{array}$ & $\begin{array}{c}1987- \\
1989\end{array}$ & $\begin{array}{l}{ }^{137} \text { Cs was mostly in } \\
\text { cap and lamellae; } \\
\text { Found in a spruce } \\
\text { and fir forest soil; } \\
\text { near } 800 \mathrm{~m} \text { asl }\end{array}$ & $\begin{array}{c}4001 \text { to } 8000 \\
\mathrm{~Bq} / \mathrm{kg} \mathrm{fw}\end{array}$ & $\begin{array}{c}\mathrm{TF}: 2.01 \text { to } \\
4.0\end{array}$ & Heinrich (1993) \\
\hline $\begin{array}{l}134 \\
137\end{array}$ & $\begin{array}{c}\text { Cortinarius } \\
\text { praestans }\end{array}$ & & $\begin{array}{l}\text { Yugo- } \\
\text { slavia }\end{array}$ & Slovenia & 1986 & $\begin{array}{l}\text { All portions; After } \\
\text { Chernobyl }\end{array}$ & $\begin{array}{c}360 \text { to } 670 \\
\mathrm{~Bq}{ }^{134,137} \mathrm{Cs} \\
/ \mathrm{kg} ; \mathrm{Mean}: \\
530 \mathrm{~Bq} \\
{ }^{134,137} \mathrm{Cs} / \mathrm{kg} \\
\end{array}$ & No data & Byrne (1988) \\
\hline 137 & $\begin{array}{l}\text { Cortinarius } \\
\text { saturninus }\end{array}$ & & Japan & Tochigi & $\begin{array}{l}\text { Sept.- } \\
\text { Dec. } \\
1990\end{array}$ & $\begin{array}{c}\text { All portions; Cleaned } \\
\text { before analyses; } \\
\text { Mycorrhizal fungi }\end{array}$ & $1700 \mathrm{~Bq} / \mathrm{kg}$ & No data & $\begin{array}{l}\text { Yoshida et al. } \\
\text { (1994); Yoshida and } \\
\text { Muramatsu (1994) }\end{array}$ \\
\hline 134 & $\begin{array}{l}\text { Cortinarius } \\
\text { saturninus }\end{array}$ & & Japan + & Tochigi & $\begin{array}{l}\text { Sept.- } \\
\text { Dec. } \\
1990\end{array}$ & $\begin{array}{l}\text { All portions; Cleaned } \\
\text { before analyses; } \\
\text { Mycorrhizal fungi }\end{array}$ & $<10 \mathrm{~Bq} / \mathrm{kg}$ & No data & $\begin{array}{l}\text { Yoshida et al. } \\
\text { (1994); Yoshida and } \\
\text { Muramatsu (1994) }\end{array}$ \\
\hline 137 & $\begin{array}{l}\text { Cortinarius } \\
\text { semi- } \\
\text { sanguineus } \\
\end{array}$ & $\begin{array}{l}\text { Poison dye } \\
\text { cort }\end{array}$ & Germany & $\begin{array}{l}\text { Southern } \\
\text { Bavaria }\end{array}$ & $\begin{array}{c}1987- \\
1990\end{array}$ & $\begin{array}{l}\text { In fruiting body } \\
\text { (assumed) }\end{array}$ & $\begin{array}{c}\text { Ratioed to } B . \\
\text { badius**:0.9 } \\
7 \text { to } 2.64 \\
\end{array}$ & No data & $\begin{array}{l}\text { Kammerer et al. } \\
(1994)\end{array}$ \\
\hline $\begin{array}{l}134 \\
137\end{array}$ & $\begin{array}{l}\text { Cortinarius } \\
\text { traganus }\end{array}$ & $\begin{array}{c}\text { Gassy } \\
\text { webcap; He- } \\
\text { Goat }\end{array}$ & $\begin{array}{l}\text { Yugo- } \\
\text { slavia }\end{array}$ & Slovenia & 1986 & $\begin{array}{l}\text { All portions; After } \\
\text { Chernobyl }\end{array}$ & $\begin{array}{c}5100 \text { to } \\
17900 \\
\mathrm{~Bq}^{134,137} \mathrm{Cs} \\
/ \mathrm{kg} ; \mathrm{Mean}: \\
12000 \mathrm{~Bq} \\
{ }_{134,137} \mathrm{Cs} / \mathrm{kg}\end{array}$ & No data & Byrne (1988) \\
\hline
\end{tabular}


WSRC-MS-2006-0422 Rev. 1

\begin{tabular}{|c|c|c|c|c|c|c|c|c|c|}
\hline 137 & $\begin{array}{l}\text { Galerina } \\
\text { mutabilis }\end{array}$ & $\begin{array}{l}\text { Scaly-veiled } \\
\text { galerina }\end{array}$ & $\begin{array}{l}\text { United } \\
\text { Kingdom }\end{array}$ & $\begin{array}{c}\text { Lake of } \\
\text { Monteith } \\
\text { and Harlech }\end{array}$ & $\begin{array}{c}1987 \\
1990\end{array}$ & $\begin{array}{l}\text { In fruiting body; } \\
\text { Saprotrophic; } \\
\text { Growing on rotting } \\
\text { wood (lignin) }\end{array}$ & $\begin{array}{c}31.9 \text { to } 261.6 \\
\mathrm{~Bq} / \mathrm{kg}\end{array}$ & No data & Watling et al. (1993) \\
\hline 137 & $\begin{array}{l}\text { Hebeloma } \\
\text { cylindro- } \\
\text { sporum }\end{array}$ & $\begin{array}{l}\text { Ectomycorrhiz } \\
\text { al fungi }\end{array}$ & Spain & Muňoveros & $\begin{array}{c}1987 \\
\text { and } \\
1990\end{array}$ & $\begin{array}{l}\text { In fruiting body; } \\
\text { Often found with } \\
\text { Pinus pinaster }\end{array}$ & $647 \mathrm{~Bq} / \mathrm{kg}$ & No data & Baeza et al. (2004) \\
\hline 137 & Hebeloma sp. & a Toadstool & Japan & Akita & $\begin{array}{c}1983- \\
1990\end{array}$ & $\begin{array}{c}\text { All portions; Cleaned } \\
\text { before analyses; } \\
\text { Mycorrhizal fungi }\end{array}$ & $16300 \mathrm{~Bq} / \mathrm{kg}$ & No data & $\begin{array}{l}\text { Yoshida et al. } \\
\text { (1994); Yoshida and } \\
\text { Muramatsu (1994) } \\
\end{array}$ \\
\hline 134 & Hebeloma sp. & a Toadstool & Japan & Akita & $\begin{array}{c}1983- \\
1990\end{array}$ & $\begin{array}{c}\text { All portions; Cleaned } \\
\text { before analyses; } \\
\text { Mycorrhizal fungi }\end{array}$ & $436 \mathrm{~Bq} / \mathrm{kg}$ & No data & $\begin{array}{l}\text { Yoshida et al. } \\
\text { (1994); Yoshida and } \\
\text { Muramatsu (1994) }\end{array}$ \\
\hline 137 & $\begin{array}{l}\text { Hydnum } \\
\text { repandum or } \\
\text { Dentinum } \\
\text { repandum }\end{array}$ & $\begin{array}{l}\text { Hedgehog; } \\
\text { Spreading } \\
\text { hedgehog }\end{array}$ & Austria & $\begin{array}{l}\text { State of } \\
\text { Styria }\end{array}$ & $\begin{array}{c}1987- \\
1989\end{array}$ & $\begin{array}{l}{ }^{137} \text { Cs was mostly in } \\
\text { lamellae and cap; } \\
\text { Found in a spruce } \\
\text { and fir forest; near } \\
800 \text { to } 1000 \mathrm{~m} \text { asl }\end{array}$ & $\begin{array}{c}16000 \text { to } \\
32000 \mathrm{~Bq} / \mathrm{kg} \\
\mathrm{wW}\end{array}$ & TF: $>4.0$ & Heinrich (1993) \\
\hline 137 & $\begin{array}{l}\text { Hydnum } \\
\text { repandum or } \\
\text { Dentinum } \\
\text { repandum }\end{array}$ & $\begin{array}{l}\text { Hedgehog; } \\
\text { Spreading } \\
\text { hedgehog }\end{array}$ & Germany & $\begin{array}{l}\text { Southern } \\
\text { Bavaria }\end{array}$ & $\begin{array}{c}1987- \\
1990\end{array}$ & $\begin{array}{l}\text { In fruiting body } \\
\text { (assumed) }\end{array}$ & $\begin{array}{l}\text { Ratioed to } B . \\
\text { badius**:1.8 } \\
7 \text { to } 6.38 \\
2420 \text { to } 1500 \\
\mathrm{~Bq} / \mathrm{kg} \mathrm{fw} \\
\end{array}$ & $\begin{array}{c}\text { TF: } 1.1 \text { to } \\
4.3 ; \\
\text { Mean: } 2.3\end{array}$ & $\begin{array}{l}\text { Kammerer et al. } \\
(1994)\end{array}$ \\
\hline 137 & $\begin{array}{l}\text { Hygrophorus } \\
\text { russula }\end{array}$ & False russula & Japan & Tochigi & $\begin{array}{l}\text { Sept.- } \\
\text { Dec. } \\
1990 \\
\end{array}$ & $\begin{array}{c}\text { All portions; Cleaned } \\
\text { before analyses; } \\
\text { Mycorrhizal fungi }\end{array}$ & $998 \mathrm{~Bq} / \mathrm{kg}$ & No data & $\begin{array}{l}\text { Yoshida et al. } \\
\text { (1994); Yoshida and } \\
\text { Muramatsu (1994) } \\
\end{array}$ \\
\hline 134 & $\begin{array}{l}\text { Hygrophorus } \\
\text { russula }\end{array}$ & False russula & Japan & Tochigi & $\begin{array}{l}\text { Sept.- } \\
\text { Dec. } \\
1990 \\
\end{array}$ & $\begin{array}{c}\text { All portions; Cleaned } \\
\text { before analyses; } \\
\text { Mycorrhizal fungi }\end{array}$ & $<9 \mathrm{~Bq} / \mathrm{kg}$ & No data & $\begin{array}{l}\text { Yoshida et al. } \\
\text { (1994); Yoshida and } \\
\text { Muramatsu (1994) }\end{array}$ \\
\hline 137 & $\begin{array}{l}\text { Hypholoma } \\
\text { fasciculare }\end{array}$ & $\begin{array}{l}\text { Sulfur tuft; } \\
\text { Sulfur tuft } \\
\text { psilocybe }\end{array}$ & $\begin{array}{l}\text { United } \\
\text { Kingdom }\end{array}$ & $\begin{array}{l}\text { Lady Wood } \\
\text { Forest, } 0.5 \\
\text { km from } \\
\text { Sellafield }\end{array}$ & $\begin{array}{l}\text { Summe } \\
\text { r } 1996\end{array}$ & $\begin{array}{l}\text { In fruiting body; } \\
\text { Cleaned before } \\
\text { analyses; From a } \\
\text { Picea sitchensis } \\
\text { woodland }\end{array}$ & $\begin{array}{c}2317 \text { to } 2622 \\
\mathrm{~Bq} / \mathrm{kg}\end{array}$ & $\begin{array}{c}\mathrm{T}_{\mathrm{ag}}: 0.09 \text { to } \\
0.1\end{array}$ & Toal et al. (2002) \\
\hline 137 & $\begin{array}{l}\text { Hypholoma } \\
\text { fasciculare }\end{array}$ & $\begin{array}{l}\text { Sulfur tuft; } \\
\text { Sulfur tuft } \\
\text { psilocybe }\end{array}$ & $\begin{array}{l}\text { United } \\
\text { Kingdom }\end{array}$ & $\begin{array}{l}\text { Lady Wood } \\
\text { Forest, } 0.5 \\
\text { km from } \\
\text { Sellafield }\end{array}$ & $\begin{array}{c}\text { Autumn } \\
1996\end{array}$ & $\begin{array}{l}\text { In fruiting body; } \\
\text { Cleaned before } \\
\text { analyses; From a } \\
\text { Picea sitchensis } \\
\text { woodland }\end{array}$ & $\begin{array}{c}781 \text { to } 1046 \\
\mathrm{~Bq} / \mathrm{kg}\end{array}$ & $\begin{array}{l}\mathrm{T}_{\mathrm{ag}}: 0.0054 \\
\quad \text { to } 0.12\end{array}$ & Toal et al. (2002) \\
\hline 137 & $\begin{array}{l}\text { Hypholoma } \\
\text { marginatum }\end{array}$ & & $\begin{array}{l}\text { United } \\
\text { Kingdom }\end{array}$ & $\begin{array}{l}\text { Lady Wood } \\
\text { Forest, } 0.5 \\
\text { km from } \\
\text { Sellafield }\end{array}$ & $\begin{array}{c}\text { Autumn } \\
1996\end{array}$ & $\begin{array}{l}\text { In fruiting body; } \\
\text { Cleaned before } \\
\text { analyses; From a } \\
\text { Picea sitchensis } \\
\text { woodland }\end{array}$ & $\begin{array}{c}781 \text { to } 1046 \\
\mathrm{~Bq} / \mathrm{kg}\end{array}$ & $\mathrm{T}_{\mathrm{ag}}: 0.039$ & Toal et al. (2002) \\
\hline 137 & Inocybe sp. & & Japan & Ibaraki & $\begin{array}{l}\text { Sept.- } \\
\text { Dec. } \\
1990\end{array}$ & $\begin{array}{c}\text { All portions; Cleaned } \\
\text { before analyses; } \\
\text { Mycorrhizal fungi }\end{array}$ & $\begin{array}{c}<38 \text { to } 887 \\
\mathrm{~Bq} / \mathrm{kg}\end{array}$ & No data & $\begin{array}{l}\text { Yoshida et al. } \\
\text { (1994); Yoshida and } \\
\text { Muramatsu (1994) }\end{array}$ \\
\hline 134 & Inocybe sp. & & Japan & Ibaraki & $\begin{array}{l}\text { Sept.- } \\
\text { Dec. } \\
1990 \\
\end{array}$ & $\begin{array}{c}\text { All portions; Cleaned } \\
\text { before analyses; } \\
\text { Mycorrhizal fungi }\end{array}$ & $\begin{array}{c}<4 \text { to } 50 \\
\mathrm{~Bq} / \mathrm{kg}\end{array}$ & No data & $\begin{array}{l}\text { Yoshida et al. } \\
\text { (1994); Yoshida and } \\
\text { Muramatsu (1994) } \\
\end{array}$ \\
\hline 137 & $\begin{array}{c}\text { Laccaria } \\
\text { amethystine }\end{array}$ & $\begin{array}{c}\text { Amethyst } \\
\text { deceiver or } \\
\text { tallogill; } \\
\text { purple laccaria }\end{array}$ & Germany & $\begin{array}{l}\text { Southern } \\
\text { Bavaria }\end{array}$ & $\begin{array}{c}1987- \\
1990\end{array}$ & $\begin{array}{l}\text { In fruiting body } \\
\text { (assumed) }\end{array}$ & $\begin{array}{l}\text { Ratioed to } B . \\
\text { badius**:0.3 } \\
\text { to } 1.96 ; 435 \\
\text { to } 5115 \\
\text { Bq } / \mathrm{kg} \mathrm{fw} \\
\end{array}$ & $\begin{array}{c}\text { TF: } 0.36 \text { to } \\
3.0 ; \\
\text { Mean: } 1.6\end{array}$ & $\begin{array}{l}\text { Kammerer et al. } \\
(1994)\end{array}$ \\
\hline $\begin{array}{l}134 \\
137\end{array}$ & $\begin{array}{l}\text { Laccaria } \\
\text { amethystine }\end{array}$ & $\begin{array}{c}\text { Amethyst } \\
\text { deceiver or } \\
\text { tallogill; } \\
\text { purple laccaria }\end{array}$ & $\begin{array}{l}\text { Yugo- } \\
\text { slavia }\end{array}$ & Slovenia & 1986 & $\begin{array}{l}\text { All portions; After } \\
\text { Chernobyl }\end{array}$ & \begin{tabular}{|c|}
12000 to \\
117000 \\
$\mathrm{~Bq}^{134,137} \mathrm{Cs} / \mathrm{k}$ \\
$\mathrm{g} ;$ Mean: \\
$52000 \mathrm{~Bq}$ \\
\end{tabular} & No data & Byrne (1988) \\
\hline
\end{tabular}


WSRC-MS-2006-0422 Rev. 1

\begin{tabular}{|c|c|c|c|c|c|c|c|c|c|}
\hline & & & & & & & ${ }^{134,137} \mathrm{Cs} / \mathrm{kg}$ & & \\
\hline 137 & $\begin{array}{c}\text { Lactarius } \\
\text { blennius }\end{array}$ & $\begin{array}{l}\text { Euro slimy } \\
\text { lactarius }\end{array}$ & $\begin{array}{l}\text { United } \\
\text { Kingdom }\end{array}$ & $\begin{array}{l}\text { Saline, } \\
\text { Dollar and } \\
\text { Cleish } \\
\end{array}$ & $\begin{array}{c}1987, \\
1990\end{array}$ & $\begin{array}{l}\text { In fruiting body; } \\
\text { Ectomycorrhizal } \\
\text { species }\end{array}$ & $\begin{array}{c}110 \text { to } 1479 \\
\mathrm{~Bq} / \mathrm{kg}\end{array}$ & No data & Watling et al. (1993) \\
\hline 137 & $\begin{array}{c}\text { Lactarius } \\
\text { corrugis }\end{array}$ & $\begin{array}{l}\text { Corrugated } \\
\text { milky cap; } \\
\text { Wrinkled } \\
\text { milkcap }\end{array}$ & Japan & Ibaraki & $\begin{array}{l}\text { Sept.- } \\
\text { Dec. } \\
1990\end{array}$ & $\begin{array}{l}\text { All portions; Cleaned } \\
\text { before analyses; } \\
\text { Mycorrhizal fungi }\end{array}$ & $2700 \mathrm{~Bq} / \mathrm{kg}$ & No data & $\begin{array}{l}\text { Yoshida et al. } \\
\text { (1994); Yoshida and } \\
\text { Muramatsu (1994) }\end{array}$ \\
\hline 134 & $\begin{array}{c}\text { Lactarius } \\
\text { corrugis }\end{array}$ & $\begin{array}{l}\text { Corrugated } \\
\text { milky cap; } \\
\text { Wrinkled } \\
\text { milkcap }\end{array}$ & Japan & Ibaraki & $\begin{array}{l}\text { Sept.- } \\
\text { Dec. } \\
1990\end{array}$ & $\begin{array}{l}\text { All portions; Cleaned } \\
\text { before analyses; } \\
\text { Mycorrhizal fungi }\end{array}$ & $51.6 \mathrm{~Bq} / \mathrm{kg}$ & No data & $\begin{array}{l}\text { Yoshida et al. } \\
\text { (1994); Yoshida and } \\
\text { Muramatsu (1994) }\end{array}$ \\
\hline 137 & $\begin{array}{l}\text { Lactarius } \\
\text { deliciosus }\end{array}$ & $\begin{array}{l}\text { Saffron } \\
\text { milkcap }\end{array}$ & Germany & $\begin{array}{l}\text { Southern } \\
\text { Bavaria }\end{array}$ & $\begin{array}{c}1987- \\
1990\end{array}$ & $\begin{array}{l}\text { In fruiting body } \\
\text { (assumed) }\end{array}$ & $\begin{array}{l}\text { Ratioed to } B \text {. } \\
\text { badius } * *: 0.1 \\
\text { to } 0.18 ; 185 \\
\text { to } 235 \mathrm{~Bq} / \mathrm{kg} \\
\text { fw }\end{array}$ & $\begin{array}{l}\text { TF: } 0.13 \text { to } \\
0.26 \\
\text { Mean: } 0.2\end{array}$ & $\begin{array}{l}\text { Kammerer et al. } \\
(1994)\end{array}$ \\
\hline 137 & $\begin{array}{l}\text { Lactarius } \\
\text { deliciosus }\end{array}$ & $\begin{array}{l}\text { Saffron } \\
\text { milkcap }\end{array}$ & Spain & La Bazagona & $\begin{array}{c}\text { Not } \\
\text { known }\end{array}$ & In fruiting body & $36 \mathrm{~Bq} / \mathrm{kg}$ & No data & Baeza et al. (2004) \\
\hline 137 & $\begin{array}{l}\text { Lactarius } \\
\text { deliciosus }\end{array}$ & $\begin{array}{l}\text { Saffron } \\
\text { milkcap }\end{array}$ & Austria & $\begin{array}{c}\text { State of } \\
\text { Styria }\end{array}$ & $\begin{array}{c}1987- \\
1989\end{array}$ & $\begin{array}{l}{ }^{137} \text { Cs was mostly in } \\
\text { lamellae and cap; } \\
\text { Found in a spruce } \\
\text { and fir forest soil; } \\
\text { near } 800 \mathrm{~m} \text { asl }\end{array}$ & $\begin{array}{l}251 \text { to } 500 \\
\mathrm{~Bq} / \mathrm{kg} \mathrm{ww}\end{array}$ & $\begin{array}{c}\text { TF: } 0 \text { to } \\
0.25\end{array}$ & Heinrich (1993) \\
\hline 137 & $\begin{array}{l}\text { Lactarius } \\
\text { deliciosus }\end{array}$ & $\begin{array}{l}\text { Saffron } \\
\text { milkcap }\end{array}$ & $\begin{array}{l}\text { United } \\
\text { Kingdom }\end{array}$ & $\begin{array}{l}\text { Lady Wood } \\
\text { Forest, } 0.5 \\
\text { km from } \\
\text { Sellafield }\end{array}$ & $\begin{array}{c}\text { Autumn } \\
1996\end{array}$ & $\begin{array}{l}\text { In fruiting body; } \\
\text { Cleaned before } \\
\text { analyses; From a } \\
\text { Picea sitchensis } \\
\text { woodland } \\
\end{array}$ & $2 \mathrm{~Bq} / \mathrm{kg}$ & $\begin{array}{c}\mathrm{T}_{\mathrm{ag}}: \\
0.000077\end{array}$ & Toal et al. (2002) \\
\hline 137 & $\begin{array}{l}\text { Lactarius } \\
\text { hepaticus }\end{array}$ & Liver milkcap & $\begin{array}{l}\text { United } \\
\text { Kingdom }\end{array}$ & $\begin{array}{l}\text { Lady Wood } \\
\text { Forest, } 0.5 \\
\text { km from } \\
\text { Sellafield }\end{array}$ & $\begin{array}{c}\text { Autumn } \\
1996\end{array}$ & $\begin{array}{c}\text { In fruiting body; } \\
\text { Cleaned before } \\
\text { analyses; From a } \\
\text { Picea sitchensis } \\
\text { woodland } \\
\end{array}$ & $\begin{array}{c}1713 \text { to } 1761 \\
\mathrm{~Bq} / \mathrm{kg}\end{array}$ & $\begin{array}{l}\mathrm{T}_{\mathrm{ag}}: 0.066 \\
\text { to } 0.068\end{array}$ & Toal et al. (2002) \\
\hline 137 & $\begin{array}{l}\text { Lactarius } \\
\text { necator }\end{array}$ & $\begin{array}{l}\text { Mutagen } \\
\text { lactarius }\end{array}$ & Ukraine & $\begin{array}{l}\text { Zhitomir } \\
\text { Region }\end{array}$ & $\begin{array}{c}1996- \\
1998\end{array}$ & $\begin{array}{c}\text { In fruiting body; } \\
\text { Collected in Scots } \\
\text { pine and birch forests }\end{array}$ & $\begin{array}{c}\text { Mean:52700 } \\
\mathrm{Bq} / \mathrm{kg}\end{array}$ & TF:4.9 & $\begin{array}{l}\text { Vinichuk and } \\
\text { Johanson (2003) }\end{array}$ \\
\hline 137 & $\begin{array}{l}\text { Lactarius } \\
\text { necator }\end{array}$ & $\begin{array}{l}\text { Mutagen } \\
\text { lactarius }\end{array}$ & Austria & $\begin{array}{l}\text { State of } \\
\text { Styria }\end{array}$ & $\begin{array}{c}1987- \\
1989\end{array}$ & $\begin{array}{l}{ }^{137} \text { Cs was mostly in } \\
\text { lamellae and cap; } \\
\text { Found in a spruce } \\
\text { and fir forestsl; near } \\
800 \text { and } 1000 \text { m asl }\end{array}$ & $\begin{array}{c}4001 \text { to } 8000 \\
\mathrm{~Bq} / \mathrm{kg} \mathrm{ww}\end{array}$ & $\begin{array}{c}\mathrm{TF}: 1.01 \text { to } \\
2\end{array}$ & Heinrich (1993) \\
\hline 137 & $\begin{array}{l}\text { Lactarius } \\
\text { piperatus }\end{array}$ & $\begin{array}{l}\text { Pepper milk- } \\
\text { cap; Peppery } \\
\text { lactarius } \\
\end{array}$ & Japan & $\begin{array}{l}\text { Akita and } \\
\text { Tochigi }\end{array}$ & $\begin{array}{l}\text { Sept.- } \\
\text { Dec. } \\
1990 \\
\end{array}$ & $\begin{array}{c}\text { All portions; Cleaned } \\
\text { before analyses; } \\
\text { Mycorrhizal fungi }\end{array}$ & $\begin{array}{l}59 \text { to } 1500 \\
\mathrm{~Bq} / \mathrm{kg}\end{array}$ & No data & $\begin{array}{l}\text { Yoshida et al. } \\
\text { (1994); Yoshida and } \\
\text { Muramatsu (1994) } \\
\end{array}$ \\
\hline 134 & $\begin{array}{l}\text { Lactarius } \\
\text { piperatus }\end{array}$ & $\begin{array}{l}\text { Pepper milk- } \\
\text { cap; Peppery } \\
\text { lactarius }\end{array}$ & Japan & $\begin{array}{l}\text { Akita and } \\
\text { Tochigi }\end{array}$ & $\begin{array}{l}\text { Sept.- } \\
\text { Dec. } \\
1990 \\
\end{array}$ & $\begin{array}{c}\text { All portions; Cleaned } \\
\text { before analyses; } \\
\text { Mycorrhizal fungi }\end{array}$ & $21.3 \mathrm{~Bq} / \mathrm{kg}$ & No data & $\begin{array}{l}\text { Yoshida et al. } \\
\text { (1994); Yoshida and } \\
\text { Muramatsu (1994) } \\
\end{array}$ \\
\hline 137 & $\begin{array}{l}\text { Lactarius } \\
\text { rufus }\end{array}$ & $\begin{array}{l}\text { Rufus milk- } \\
\text { cap; Red hot } \\
\text { milk cap or } \\
\text { lactarius }\end{array}$ & $\begin{array}{l}\text { United } \\
\text { Kingdom }\end{array}$ & $\begin{array}{c}\text { Not } \\
\text { specified }\end{array}$ & $1980 \mathrm{~s}$ & $\begin{array}{c}\text { In fruiting body; } \\
\text { Additionally, based } \\
\text { on the }{ }^{134 / 137} \text { Cs ratio, } \\
90 \% \text { of the soil }{ }^{137} \mathrm{Cs} \\
\text { was deposited prior } \\
\text { to Chernobyl }\end{array}$ & $3900 \mathrm{~Bq} / \mathrm{kg}$ & No data & $\begin{array}{l}\text { Cawes and Horrill } \\
\text { (1986); Dighton and } \\
\text { Horrill (1988) as } \\
\text { cited in Simkiss et al. } \\
\text { (1993) }\end{array}$ \\
\hline 137 & $\begin{array}{l}\text { Lactarius } \\
\quad \text { rufus }\end{array}$ & $\begin{array}{l}\text { Rufus milk- } \\
\text { cap; Red hot } \\
\text { milk cap or } \\
\text { lactarius }\end{array}$ & Germany & $\begin{array}{l}\text { Southern } \\
\text { Bavaria }\end{array}$ & $\begin{array}{c}1987- \\
1990\end{array}$ & $\begin{array}{l}\text { In fruiting body } \\
\text { (assumed) }\end{array}$ & $\begin{array}{l}\text { Ratioed to } B \text {. } \\
\text { badius } * *: \\
1.09 \text { to } 2.04\end{array}$ & No data & $\begin{array}{l}\text { Kammerer et al. } \\
\text { (1994) }\end{array}$ \\
\hline
\end{tabular}


WSRC-MS-2006-0422 Rev. 1

\begin{tabular}{|c|c|c|c|c|c|c|c|c|c|}
\hline 137 & $\begin{array}{l}\text { Lactarius } \\
\quad \text { rufus }\end{array}$ & $\begin{array}{l}\text { Rufus milk- } \\
\text { cap; Red hot } \\
\text { milk cap or } \\
\text { lactarius }\end{array}$ & $\begin{array}{l}\text { United } \\
\text { Kingdom }\end{array}$ & $\begin{array}{l}\text { Lady Wood } \\
\text { Forest, } 0.5 \\
\text { km from } \\
\text { Sellafield }\end{array}$ & $\begin{array}{c}\text { Autumn } \\
1996\end{array}$ & $\begin{array}{l}\text { In fruiting body; } \\
\text { Cleaned before } \\
\text { analyses; From a } \\
\text { Picea sitchensis } \\
\text { woodland }\end{array}$ & $\begin{array}{c}566 \text { to } 1961 \\
\mathrm{~Bq} / \mathrm{kg}\end{array}$ & $\begin{array}{l}\mathrm{T}_{\mathrm{ag}}: 0.022 \\
\text { to } 0.076\end{array}$ & Toal et al. (2002) \\
\hline 137 & $\begin{array}{l}\text { Lactarius } \\
\quad \text { rufus }\end{array}$ & $\begin{array}{l}\text { Rufus milk- } \\
\text { cap; Red hot } \\
\text { milk cap or } \\
\text { lactarius }\end{array}$ & Austria & $\begin{array}{l}\text { State of } \\
\text { Styria }\end{array}$ & $\begin{array}{l}1987- \\
1989\end{array}$ & $\begin{array}{l}{ }^{137} \text { Cs was mostly in } \\
\text { lamellae and cap; } \\
\text { Found in a spruce } \\
\text { and fir forest soil; } \\
\text { near } 800 \text { and } 1000 \mathrm{~m} \\
\text { asl }\end{array}$ & $\begin{array}{c}8001 \text { to } \\
16000 \mathrm{~Bq} / \mathrm{kg} \\
\text { ww }\end{array}$ & $\begin{array}{c}\mathrm{TF}: 1.01 \text { to } \\
2\end{array}$ & Heinrich (1993) \\
\hline 137 & $\begin{array}{l}\text { Lactarius } \\
\text { vellereus }\end{array}$ & $\begin{array}{l}\text { Fleecy } \\
\text { lactarius }\end{array}$ & $\begin{array}{l}\text { United } \\
\text { Kingdom }\end{array}$ & $\begin{array}{c}\text { Saline, } \\
\text { Powmill and } \\
\text { Loch Ard }\end{array}$ & $\begin{array}{l}1987 \\
1990\end{array}$ & $\begin{array}{l}\text { In fruiting body; } \\
\text { Ectomycorrhizal } \\
\text { species }\end{array}$ & $\begin{array}{c}117 \text { to } 880 \\
\mathrm{~Bq} / \mathrm{kg}\end{array}$ & No data & Watling et al. (1993) \\
\hline 137 & $\begin{array}{l}\text { Lactarius } \\
\text { vellereus }\end{array}$ & $\begin{array}{l}\text { Fleecy } \\
\text { lactarius }\end{array}$ & Austria & $\begin{array}{l}\text { State of } \\
\text { Styria }\end{array}$ & $\begin{array}{l}1987- \\
1989\end{array}$ & $\begin{array}{l}\text { Mostly in lamellae } \\
\text { and cap; Found in a } \\
\text { spruce and fir forest } \\
\text { soil; near } 800 \mathrm{~m} \text { asl }\end{array}$ & $\begin{array}{c}1001 \text { to } 2000 \\
\mathrm{~Bq} / \mathrm{kg} \mathrm{ww}\end{array}$ & $\begin{array}{c}\mathrm{TF}: 0.25 \text { to } \\
0.5\end{array}$ & Heinrich (1993) \\
\hline 137 & $\begin{array}{l}\text { Lactarius } \\
\text { viestus }\end{array}$ & Grey milk-cap & Ukraine & $\begin{array}{l}\text { Zhitomir } \\
\text { Region }\end{array}$ & $\begin{array}{l}1996- \\
1998\end{array}$ & $\begin{array}{l}\text { In fruiting body; } \\
\text { From Scots pine and } \\
\text { birch forests }\end{array}$ & $\begin{array}{c}\text { Mean:56500 } \\
\mathrm{Bq} / \mathrm{kg}\end{array}$ & TF:23.7 & $\begin{array}{l}\text { Vinichuk and } \\
\text { Johanson (2003) }\end{array}$ \\
\hline 137 & $\begin{array}{c}\text { (Macro)Lepiot } \\
\text { a procera }\end{array}$ & $\begin{array}{l}\text { (Shaggy) } \\
\text { parasol } \\
\text { mushroom }\end{array}$ & Italy & $\begin{array}{l}\text { Northeastern } \\
\text { Portion }\end{array}$ & 1986 & $\begin{array}{l}\text { All portions; After } \\
\text { Chernobyl }\end{array}$ & $\begin{array}{c}221 \text { to } 2279 \\
\mathrm{~Bq} / \mathrm{kg}\end{array}$ & $\begin{array}{c}\text { TF: } 0.018 \\
\text { to } 0.11\end{array}$ & $\begin{array}{l}\text { Battiston et al. } \\
(1989)\end{array}$ \\
\hline 137 & $\begin{array}{c}\text { (Macro)Lepiot } \\
\text { a procera }\end{array}$ & $\begin{array}{c}\text { (Shaggy) } \\
\text { parasol } \\
\text { mushroom }\end{array}$ & Italy & $\begin{array}{l}\text { Northeastern } \\
\text { Portion }\end{array}$ & 1986 & $\begin{array}{l}\text { All portions; Does } \\
\text { not include }{ }^{137} \mathrm{Cs} \\
\text { from Chernobyl }\end{array}$ & $\begin{array}{c}53 \text { to } 368 \\
\mathrm{~Bq} / \mathrm{kg}\end{array}$ & No data & $\begin{array}{l}\text { Battiston et al. } \\
(1989)\end{array}$ \\
\hline 134 & $\begin{array}{l}\text { (Macro)Lepiot } \\
\text { a procera }\end{array}$ & $\begin{array}{l}\text { (Shaggy) } \\
\text { parasol } \\
\text { mushroom }\end{array}$ & Italy & $\begin{array}{l}\text { Northeastern } \\
\text { Portion }\end{array}$ & 1986 & $\begin{array}{l}\text { All portions; Does } \\
\text { not include }{ }^{137} \mathrm{Cs} \\
\text { from Chernobyl }\end{array}$ & $\begin{array}{c}116 \text { to } 263 \\
\mathrm{~Bq} / \mathrm{kg}\end{array}$ & TF:0.056 & $\begin{array}{l}\text { Battiston et al. } \\
(1989)\end{array}$ \\
\hline 137 & $\begin{array}{c}\text { (Macro)Lepiot } \\
\text { a procera }\end{array}$ & $\begin{array}{l}\text { (Shaggy) } \\
\text { parasol } \\
\text { mushroom }\end{array}$ & Austria & $\begin{array}{l}\text { State of } \\
\text { Styria }\end{array}$ & $\begin{array}{l}1987- \\
1989\end{array}$ & $\begin{array}{l}{ }^{137} \text { Cs was mostly in } \\
\text { lamellae and cap; } \\
\text { Found in a spruce } \\
\text { and fir forest soil; } \\
500 \text { and } 800 \mathrm{~m} \text { asl }\end{array}$ & $\begin{array}{c}1 \text { to } 250 \\
\mathrm{~Bq} / \mathrm{kg} \text { ww }\end{array}$ & $\begin{array}{l}\text { TF:0.019 } \\
\text { to } 0.053\end{array}$ & Heinrich (1993) \\
\hline 137 & $\begin{array}{c}\text { (Macro)Lepiot } \\
\text { a procera }\end{array}$ & $\begin{array}{l}\text { (Shaggy) } \\
\text { parasol } \\
\text { mushroom }\end{array}$ & $\begin{array}{l}\text { United } \\
\text { Kingdom }\end{array}$ & $\begin{array}{l}\text { Lady Wood } \\
\text { Forest, } 0.5 \\
\text { km from } \\
\text { Sellafield }\end{array}$ & $\begin{array}{c}\text { Autumn } \\
1996\end{array}$ & $\begin{array}{l}\text { In fruiting body; } \\
\text { Cleaned before } \\
\text { analyses; From a } \\
\text { Picea sitchensis } \\
\text { woodland } \\
\end{array}$ & $14 \mathrm{~Bq} / \mathrm{kg}$ & $\begin{array}{c}\mathrm{T}_{\mathrm{ag}}: \\
0.00056\end{array}$ & Toal et al. (2002) \\
\hline 137 & Lepista irina & $\begin{array}{l}\text { Pungent false } \\
\text { blewit }\end{array}$ & Germany & $\begin{array}{l}\text { Southern } \\
\text { Bavaria }\end{array}$ & $\begin{array}{c}1987- \\
1990\end{array}$ & $\begin{array}{l}\text { In fruiting body } \\
\text { (assumed) }\end{array}$ & $\begin{array}{l}\text { Ratioed to } B . \\
\text { badius**:0.0 } \\
1 \text { to } 0.11 ; 13 \\
\text { to } 2110 \\
\mathrm{~Bq} / \mathrm{kg} \mathrm{fw} \\
\end{array}$ & $\begin{array}{c}\text { TF: } 0.003 \\
\text { to } 2.2 ; \\
\text { Mean:0.84 }\end{array}$ & $\begin{array}{l}\text { Kammerer et al. } \\
(1994)\end{array}$ \\
\hline 137 & Lepista nuda & $\begin{array}{l}\text { Wood or true } \\
\text { blewit }\end{array}$ & Germany & $\begin{array}{l}\text { Southern } \\
\text { Bavaria }\end{array}$ & $\begin{array}{c}1987- \\
1990\end{array}$ & $\begin{array}{l}\text { In fruiting body } \\
\text { (assumed) }\end{array}$ & $\begin{array}{l}\text { Ratioed to } B \text {. } \\
\text { badius } * * \text { : } \\
0.02 \text { to } 0.07 \\
32 \text { to } 285 \\
\mathrm{~Bq} / \mathrm{kg} \mathrm{fw}\end{array}$ & $\begin{array}{c}\text { TF: } 0.2 \text { to } \\
0.33 ; \\
\text { Mean: } 0.14\end{array}$ & $\begin{array}{l}\text { Kammerer et al. } \\
(1994)\end{array}$ \\
\hline 137 & Lepista nuda & $\begin{array}{l}\text { Wood or true } \\
\text { blewit }\end{array}$ & Japan & Tochigi & $\begin{array}{l}\text { Sept.- } \\
\text { Dec. } \\
1990\end{array}$ & $\begin{array}{c}\text { All portions; Cleaned } \\
\text { before analyses; } \\
\text { Saprotrophic fungi }\end{array}$ & $\begin{array}{l}\text { Up to } 1990 \\
\mathrm{~Bq} / \mathrm{kg}\end{array}$ & No data & $\begin{array}{l}\text { Yoshida et al. } \\
\text { (1994); Yoshida and } \\
\text { Muramatsu (1994) }\end{array}$ \\
\hline 134 & Lepista nuda & $\begin{array}{l}\text { Wood or true } \\
\text { blewit }\end{array}$ & Japan & Tochigi & $\begin{array}{l}\text { Sept.- } \\
\text { Dec. } \\
1990 \\
\end{array}$ & $\begin{array}{c}\text { All portions; Cleaned } \\
\text { before analyses; } \\
\text { Saprotrophic fungi }\end{array}$ & $13.1 \mathrm{~Bq} / \mathrm{kg}$ & No data & $\begin{array}{l}\text { Yoshida et al. } \\
\text { (1994); Yoshida and } \\
\text { Muramatsu (1994) } \\
\end{array}$ \\
\hline 137 & $\begin{array}{l}\text { Lycoperdon } \\
\text { perlatum }\end{array}$ & Puffball & $\begin{array}{l}\text { Yugo- } \\
\text { slavia }\end{array}$ & Slovenia & 1986 & All; After Chernobyl & $\begin{array}{l}300 \text { to } 310 \\
\mathrm{~Bq} / \mathrm{kg}\end{array}$ & No data & Byrne (1988) \\
\hline 134 & $\begin{array}{l}\text { Lycoperdon } \\
\text { perlatum }\end{array}$ & Puffball & $\begin{array}{l}\text { Yugo- } \\
\text { slavia }\end{array}$ & Slovenia & 1986 & All; After Chernobyl & $\begin{array}{c}130 \text { to } 140 \\
\mathrm{~Bq} / \mathrm{kg}\end{array}$ & No data & Byrne (1988) \\
\hline 137 & Lycoperdon & Stump & United & Lady Wood & Autumn & In fruiting body; & 2481 to 4655 & & Toal et al. (2002) \\
\hline
\end{tabular}




\begin{tabular}{|c|c|c|c|c|c|c|c|c|c|}
\hline & pyriforme & puffball & Kingdom & $\begin{array}{l}\text { Forest, } 0.5 \\
\text { km from } \\
\text { Sellafield }\end{array}$ & 1996 & $\begin{array}{l}\text { Cleaned before } \\
\text { analyses; From a } \\
\text { Picea sitchensis } \\
\text { woodland }\end{array}$ & $\mathrm{Bq} / \mathrm{kg}$ & $\mathrm{T}_{\mathrm{ag}}: 0.12$ & \\
\hline 137 & $\begin{array}{c}\text { Mycena } \\
\text { galericulata }\end{array}$ & $\begin{array}{l}\text { Common } \\
\text { tufted, Rosy- } \\
\text { gill fairy hel- } \\
\text { met or bonnet } \\
\text { mycena }\end{array}$ & $\begin{array}{l}\text { United } \\
\text { Kingdom }\end{array}$ & $\begin{array}{l}\text { Lady Wood } \\
\text { Forest, } 0.5 \\
\text { km from } \\
\text { Sellafield }\end{array}$ & $\begin{array}{c}\text { Autumn } \\
1996\end{array}$ & $\begin{array}{l}\text { In fruiting body; } \\
\text { Cleaned before } \\
\text { analyses; From a } \\
\text { Picea sitchensis } \\
\text { woodland }\end{array}$ & $1320 \mathrm{~Bq} / \mathrm{kg}$ & $\mathrm{T}_{\mathrm{ag}}: 0.12$ & Toal et al. (2002) \\
\hline 137 & $\begin{array}{c}\text { Mycena } \\
\text { galericulata }\end{array}$ & $\begin{array}{l}\text { Common } \\
\text { tufted, Rosy- } \\
\text { gill fairy hel- } \\
\text { met or bonnet } \\
\text { mycena } \\
\end{array}$ & $\begin{array}{l}\text { United } \\
\text { Kingdom }\end{array}$ & $\begin{array}{l}\text { Lady Wood } \\
\text { Forest, } 0.5 \\
\text { km from } \\
\text { Sellafield }\end{array}$ & $\begin{array}{c}\text { Autumn } \\
1996\end{array}$ & $\begin{array}{l}\text { In fruiting body; } \\
\text { Cleaned before } \\
\text { analyses; From a } \\
\text { Picea sitchensis } \\
\text { woodland }\end{array}$ & $\begin{array}{c}\text { Mean:3213 } \\
\mathrm{Bq} / \mathrm{kg}\end{array}$ & $\begin{array}{l}\mathrm{T}_{\mathrm{ag}}: 0.096 \\
\text { to } 0.18\end{array}$ & Toal et al. (2002) \\
\hline 137 & $\begin{array}{c}\text { Mycena } \\
\text { galericulata }\end{array}$ & $\begin{array}{l}\text { Common } \\
\text { tufted, Rosy- } \\
\text { gill fairy hel- } \\
\text { met or bonnet } \\
\text { mycena }\end{array}$ & $\begin{array}{l}\text { United } \\
\text { Kingdom }\end{array}$ & $\begin{array}{l}\text { Lady Wood } \\
\text { Forest, } 0.5 \\
\text { km from } \\
\text { Sellafield }\end{array}$ & $\begin{array}{l}\text { Winter } \\
1996- \\
1997\end{array}$ & $\begin{array}{l}\text { In fruiting body; } \\
\text { Cleaned before } \\
\text { analyses; From a } \\
\text { Picea sitchensis } \\
\text { woodland }\end{array}$ & $2984 \mathrm{~Bq} / \mathrm{kg}$ & $\mathrm{T}_{\mathrm{ag}}: 0.21$ & Toal et al. (2002) \\
\hline 137 & $\begin{array}{l}\text { Naematoloma } \\
\text { subleteritium }\end{array}$ & Brick cap & Japan & $\begin{array}{c}\text { Iwate, } \\
\text { Gunma and } \\
\text { Akita } \\
\end{array}$ & $\begin{array}{l}\text { Sept.- } \\
\text { Dec. } \\
1990 \\
\end{array}$ & $\begin{array}{l}\text { All portions; Cleaned } \\
\text { before analyses; } \\
\text { Saprotrophic fungi }\end{array}$ & $\begin{array}{l}\text { Up to } 151 \\
\mathrm{~Bq} / \mathrm{kg}\end{array}$ & No data & $\begin{array}{l}\text { Yoshida et al. } \\
\text { (1994); Yoshida and } \\
\text { Muramatsu (1994) }\end{array}$ \\
\hline 134 & $\begin{array}{l}\text { Naematoloma } \\
\text { subleteritium }\end{array}$ & Brick cap & Japan & $\begin{array}{c}\text { Iwate, } \\
\text { Gunma and } \\
\text { Akita }\end{array}$ & $\begin{array}{l}\text { Sept.- } \\
\text { Dec. } \\
1990 \\
\end{array}$ & $\begin{array}{l}\text { All portions; Cleaned } \\
\text { before analyses; } \\
\text { Saprotrophic fungi }\end{array}$ & $<6 \mathrm{~Bq} / \mathrm{kg}$ & No data & $\begin{array}{l}\text { Yoshida et al. } \\
\text { (1994); Yoshida and } \\
\text { Muramatsu (1994) }\end{array}$ \\
\hline 137 & $\begin{array}{l}\text { Paxillus } \\
\text { involutus }\end{array}$ & $\begin{array}{l}\text { Brown roll- } \\
\text { rim; Poison } \\
\text { paxillus; Nak- } \\
\text { ed brimcap }\end{array}$ & Germany & $\begin{array}{l}\text { Western } \\
\text { Germany }\end{array}$ & $\begin{array}{l}1963- \\
1966\end{array}$ & $\begin{array}{l}\text { In fruiting body } \\
\text { (assumed); Soil } \\
\text { cleaned off prior to } \\
\text { analysis }\end{array}$ & $\begin{array}{l}216 \text { to } 1097 \\
\mathrm{~Bq} / \mathrm{kg} \mathrm{fw}\end{array}$ & No data & Greuter (1971) \\
\hline 137 & $\begin{array}{l}\text { Paxillus } \\
\text { involutus }\end{array}$ & $\begin{array}{l}\text { Brown roll- } \\
\text { rim; Poison } \\
\text { paxillus; Nak- } \\
\text { ed brimcap }\end{array}$ & Europe & $\begin{array}{c}\text { Sweden, } \\
\text { Switzerland, } \\
\text { Italy, Austria } \\
\text { and Finland }\end{array}$ & 1974 & In fruiting body & $\begin{array}{c}\text { Mean: 1481 } \\
\text { Bq/kg }\end{array}$ & No data & Haselwandter (1978) \\
\hline 137 & $\begin{array}{l}\text { Paxillus } \\
\text { involutus }\end{array}$ & $\begin{array}{l}\text { Brown roll- } \\
\text { rim; Poison } \\
\text { paxillus; Nak- } \\
\text { ed brimcap }\end{array}$ & Germany & $\begin{array}{l}\text { Southern } \\
\text { Bavaria }\end{array}$ & $\begin{array}{c}1987- \\
1990\end{array}$ & $\begin{array}{l}\text { In fruiting body } \\
\text { (assumed) }\end{array}$ & $\begin{array}{l}\text { Ratioed to } B . \\
\text { badius } * *: \\
1.24 \text { to } 2.43\end{array}$ & No data & $\begin{array}{l}\text { Kammerer et al. } \\
\text { (1994) }\end{array}$ \\
\hline 137 & $\begin{array}{l}\text { Paxillus } \\
\text { involutus }\end{array}$ & $\begin{array}{l}\text { Brown roll- } \\
\text { rim; Poison } \\
\text { paxillus; Nak- } \\
\text { ed brimcap }\end{array}$ & Ukraine & $\begin{array}{l}\text { Zhitomir } \\
\text { region }\end{array}$ & $\begin{array}{l}1996- \\
1998\end{array}$ & $\begin{array}{l}\text { In fruiting body; } \\
\text { From Scots pine and } \\
\text { birch forests }\end{array}$ & $\begin{array}{c}\text { Mean:86210 } \\
0 \mathrm{~Bq} / \mathrm{kg}\end{array}$ & $\mathrm{TF}: 21.6$ & $\begin{array}{l}\text { Vinichuk and } \\
\text { Johanson (2003) }\end{array}$ \\
\hline 137 & $\begin{array}{l}\text { Paxillus } \\
\text { involutus }\end{array}$ & $\begin{array}{l}\text { Brown roll- } \\
\text { rim; Poison } \\
\text { paxillus; Nak- } \\
\text { ed brimcap }\end{array}$ & $\begin{array}{l}\text { United } \\
\text { Kingdom }\end{array}$ & $\begin{array}{l}\text { Prescot and } \\
\text { Rhosesmor }\end{array}$ & $\begin{array}{l}1987 \\
1990\end{array}$ & $\begin{array}{l}\text { In fruiting body; } \\
\text { Ectomycorrhizal } \\
\text { species }\end{array}$ & $\begin{array}{c}\text { Below } \\
\text { detection } \\
\text { and } 68.4 \\
\mathrm{~Bq} / \mathrm{kg}\end{array}$ & No data & Watling et al. (1993) \\
\hline 137 & $\begin{array}{l}\text { Paxillus } \\
\text { involutus }\end{array}$ & $\begin{array}{l}\text { Brown roll- } \\
\text { rim; Poison } \\
\text { paxillus; Nak- } \\
\text { ed brimcap }\end{array}$ & Austria & $\begin{array}{l}\text { State of } \\
\text { Styria }\end{array}$ & $\begin{array}{l}1987- \\
1989\end{array}$ & $\begin{array}{l}{ }^{137} \text { Cs was mostly in } \\
\text { lamellae and cap; } \\
\text { Found in a spruce } \\
\text { and fir forest soil; } \\
500 \text { to } 1000 \mathrm{~m} \text { asl }\end{array}$ & $\begin{array}{c}8001 \text { to } \\
16000 \mathrm{~Bq} / \mathrm{kg} \\
\mathrm{ww}\end{array}$ & $\begin{array}{c}\mathrm{TF}: 2.01 \text { to } \\
4.0\end{array}$ & Heinrich (1993) \\
\hline 137 & $\begin{array}{l}\text { Panellus } \\
\text { serotinus }\end{array}$ & $\begin{array}{l}\text { Fall oyster } \\
\text { mushroom }\end{array}$ & Japan & $\begin{array}{l}\text { Nagano and } \\
\text { Akita }\end{array}$ & $\begin{array}{l}\text { Sept.- } \\
\text { Dec. } \\
1990\end{array}$ & $\begin{array}{l}\text { All portions; Cleaned } \\
\text { before analyses; } \\
\text { Saprotrophic fungi }\end{array}$ & $\begin{array}{l}\text { Up to } 462 \\
\mathrm{~Bq} / \mathrm{kg}\end{array}$ & No data & $\begin{array}{l}\text { Yoshida et al. } \\
\text { (1994); Yoshida and } \\
\text { Muramatsu (1994) }\end{array}$ \\
\hline 134 & $\begin{array}{l}\text { Panellus } \\
\text { serotinus }\end{array}$ & $\begin{array}{l}\text { Fall oyster } \\
\text { mushroom }\end{array}$ & Japan & $\begin{array}{l}\text { Nagano and } \\
\text { Akita }\end{array}$ & $\begin{array}{l}\text { Sept.- } \\
\text { Dec. } \\
1990\end{array}$ & $\begin{array}{l}\text { All portions; Cleaned } \\
\text { before analyses; } \\
\text { Saprotrophic fungi }\end{array}$ & $<7 \mathrm{~Bq} / \mathrm{kg}$ & No data & $\begin{array}{l}\text { Yoshida et al. } \\
\text { (1994); Yoshida and } \\
\text { Muramatsu (1994) }\end{array}$ \\
\hline 137 & $\begin{array}{c}\text { Pholiota } \\
\text { nameko }\end{array}$ & $\begin{array}{l}\text { Nameko } \\
\text { mushroom }\end{array}$ & Japan & $\begin{array}{c}\text { Nagano, } \\
\text { Iwate, Ohita } \\
\text { and Akita }\end{array}$ & $\begin{array}{l}\text { Sept.- } \\
\text { Dec. } \\
1990\end{array}$ & $\begin{array}{l}\text { All portions; Cleaned } \\
\text { before analyses; } \\
\text { Saprotrophic fungi }\end{array}$ & $\begin{array}{l}50 \text { to } 288 \\
\mathrm{~Bq} / \mathrm{kg}\end{array}$ & No data & $\begin{array}{l}\text { Yoshida et al. } \\
\text { (1994); Yoshida and } \\
\text { Muramatsu (1994) }\end{array}$ \\
\hline 134 & $\begin{array}{l}\text { Pholiota } \\
\text { nameko }\end{array}$ & $\begin{array}{l}\text { Nameko } \\
\text { mushroom }\end{array}$ & Japan & $\begin{array}{c}\text { Nagano, } \\
\text { Iwate, Ohita }\end{array}$ & $\begin{array}{l}\text { Sept.- } \\
\text { Dec. }\end{array}$ & $\begin{array}{l}\text { All portions; Cleaned } \\
\text { before analyses; }\end{array}$ & $<6 \mathrm{~Bq} / \mathrm{kg}$ & No data & $\begin{array}{l}\text { Yoshida et al. } \\
\text { (1994); Yoshida and }\end{array}$ \\
\hline
\end{tabular}


WSRC-MS-2006-0422 Rev. 1

\begin{tabular}{|c|c|c|c|c|c|c|c|c|c|}
\hline & & & & and Akita & 1990 & Saprotrophic fungi & & & Muramatsu (1994) \\
\hline 137 & $\begin{array}{l}\text { Rhodophyllus } \\
\text { crassipes or } \\
\text { Entoloma } \\
\text { sarcopus }\end{array}$ & Pinkgill & Japan & Tochigi & $\begin{array}{l}\text { Sept.- } \\
\text { Dec. } \\
1990\end{array}$ & $\begin{array}{c}\text { All portions; Cleaned } \\
\text { before analyses; } \\
\text { Edible; Mycorrhizal } \\
\text { fungi }\end{array}$ & $2050 \mathrm{~Bq} / \mathrm{kg}$ & No data & $\begin{array}{l}\text { Yoshida et al. } \\
\text { (1994); Yoshida and } \\
\text { Muramatsu (1994) }\end{array}$ \\
\hline 134 & $\begin{array}{c}\text { Rhodophyllus } \\
\text { crassipes or } \\
\text { Entoloma } \\
\text { sarcopus } \\
\end{array}$ & Pinkgill & Japan & Tochigi & $\begin{array}{l}\text { Sept.- } \\
\text { Dec. } \\
1990\end{array}$ & $\begin{array}{c}\text { All portions; Cleaned } \\
\text { before analyses; } \\
\text { Edible; Mycorrhizal } \\
\text { fungi } \\
\end{array}$ & $<11 \mathrm{~Bq} / \mathrm{kg}$ & No data & $\begin{array}{l}\text { Yoshida et al. } \\
\text { (1994); Yoshida and } \\
\text { Muramatsu (1994) }\end{array}$ \\
\hline 137 & $\begin{array}{l}\text { Rhodophyllus } \\
\text { rhodopolius or } \\
\text { Entoloma } \\
\text { rhodopolium }\end{array}$ & $\begin{array}{l}\text { Beech woods } \\
\text { entoloma }\end{array}$ & Japan & $\begin{array}{l}\text { Akita and } \\
\text { Tochigi }\end{array}$ & $\begin{array}{l}\text { Sept.- } \\
\text { Dec. } \\
1990\end{array}$ & $\begin{array}{c}\text { All portions; Cleaned } \\
\text { before analyses; } \\
\text { Poisonous; } \\
\text { Mycorrhizal fungi }\end{array}$ & $\begin{array}{c}149 \text { to } 2210 \\
\mathrm{~Bq} / \mathrm{kg}\end{array}$ & No data & $\begin{array}{l}\text { Yoshida et al. } \\
\text { (1994); Yoshida and } \\
\text { Muramatsu (1994) }\end{array}$ \\
\hline 134 & $\begin{array}{l}\text { Rhodophyllus } \\
\text { rhodopolius or } \\
\text { Entoloma } \\
\text { rhodopolium }\end{array}$ & $\begin{array}{l}\text { Beech woods } \\
\text { entoloma }\end{array}$ & Japan & $\begin{array}{c}\text { Akita and } \\
\text { Tochigi }\end{array}$ & $\begin{array}{l}\text { Sept.- } \\
\text { Dec. } \\
1990\end{array}$ & $\begin{array}{c}\text { All portions; Cleaned } \\
\text { before analyses; } \\
\text { Poisonous; } \\
\text { Mycorrhizal fungi }\end{array}$ & $25 \mathrm{~Bq} / \mathrm{kg}$ & No data & $\begin{array}{l}\text { Yoshida et al. } \\
\text { (1994); Yoshida and } \\
\text { Muramatsu (1994) }\end{array}$ \\
\hline 137 & $\begin{array}{l}\text { Rhodophyllus } \\
\text { rhodopolius or } \\
\text { Entoloma } \\
\text { rhodopolium }\end{array}$ & $\begin{array}{l}\text { Beech woods } \\
\text { entoloma }\end{array}$ & $\begin{array}{c}\text { United } \\
\text { Kingdom }\end{array}$ & $\begin{array}{l}\text { Lady Wood } \\
\text { Forest, } 0.5 \\
\text { km from } \\
\text { Sellafield }\end{array}$ & $\begin{array}{l}\text { Sum- } \\
\text { mer } \\
1996\end{array}$ & $\begin{array}{l}\text { In fruiting body; } \\
\text { Cleaned before } \\
\text { analyses; From a } \\
\text { Picea sitchensis } \\
\text { woodland }\end{array}$ & $\begin{array}{c}163 \text { to } \\
388 \mathrm{~Bq} / \mathrm{kg}\end{array}$ & $\begin{array}{c}\mathrm{T}_{\mathrm{ag}}: 0.0063 \\
\text { to } 0.015\end{array}$ & Toal et al. (2002) \\
\hline 137 & $\begin{array}{c}\text { Russula } \\
\text { aeruginea }\end{array}$ & $\begin{array}{l}\text { Tacky green } \\
\text { russula }\end{array}$ & $\begin{array}{l}\text { United } \\
\text { Kingdom }\end{array}$ & $\begin{array}{l}\text { Saline and } \\
\text { Cleish }\end{array}$ & $\begin{array}{c}1987, \\
1990\end{array}$ & $\begin{array}{l}\text { In fruiting body; } \\
\text { Ectomycorrhizal } \\
\text { species }\end{array}$ & $\begin{array}{c}414.7 \text { to } \\
832.5 \mathrm{~Bq} / \mathrm{kg}\end{array}$ & No data & Watling et al. (1993) \\
\hline 137 & $\begin{array}{c}\text { Russula } \\
\text { mairei }\end{array}$ & $\begin{array}{l}\text { Euro emetic } \\
\text { russula }\end{array}$ & $\begin{array}{c}\text { United } \\
\text { Kingdom }\end{array}$ & $\begin{array}{l}\text { Saline and } \\
\text { Cleish }\end{array}$ & $\begin{array}{c}1987 \\
1990\end{array}$ & $\begin{array}{l}\text { In fruiting body; } \\
\text { Ectomycorrhizal } \\
\text { species }\end{array}$ & $\begin{array}{c}251.6 \text { to } \\
1011.3 \\
\mathrm{~Bq} / \mathrm{kg}\end{array}$ & No data & Watling et al. (1993) \\
\hline 137 & $\begin{array}{l}\text { Russula } \\
\text { nigricans }\end{array}$ & $\begin{array}{c}\text { Blackening } \\
\text { russula }\end{array}$ & $\begin{array}{c}\text { United } \\
\text { Kingdom }\end{array}$ & $\begin{array}{l}\text { Linn Park } \\
\text { and Dollar }\end{array}$ & $\begin{array}{l}1987 \\
1990\end{array}$ & $\begin{array}{c}\text { In fruiting body; } \\
\text { Ectomycorrhizal } \\
\text { species }\end{array}$ & $\begin{array}{c}107.4 \text { to } 395 \\
\mathrm{~Bq} / \mathrm{kg}\end{array}$ & No data & Watling et al. (1993) \\
\hline 137 & $\begin{array}{l}\text { Russula } \\
\text { nigricans }\end{array}$ & $\begin{array}{l}\text { Blackening } \\
\text { russula }\end{array}$ & Japan & $\begin{array}{c}\text { Nagano and } \\
\text { Akita }\end{array}$ & $\begin{array}{l}\text { Sept.- } \\
\text { Dec. } \\
1990 \\
\end{array}$ & $\begin{array}{c}\text { All portions; Cleaned } \\
\text { before analyses; } \\
\text { Mycorrhizal fungi }\end{array}$ & $107 \mathrm{~Bq} / \mathrm{kg}$ & No data & $\begin{array}{l}\text { Yoshida et al. } \\
\text { (1994); Yoshida and } \\
\text { Muramatsu (1994) } \\
\end{array}$ \\
\hline 134 & $\begin{array}{l}\text { Russula } \\
\text { nigricans }\end{array}$ & $\begin{array}{l}\text { Blackening } \\
\text { russula }\end{array}$ & Japan & $\begin{array}{c}\text { Nagano and } \\
\text { Akita }\end{array}$ & $\begin{array}{l}\text { Sept.- } \\
\text { Dec. } \\
1990\end{array}$ & $\begin{array}{c}\text { All portions; Cleaned } \\
\text { before analyses; } \\
\text { Mycorrhizal fungi }\end{array}$ & $<8 \mathrm{~Bq} / \mathrm{kg}$ & No data & $\begin{array}{l}\text { Yoshida et al. } \\
\text { (1994); Yoshida and } \\
\text { Muramatsu (1994) }\end{array}$ \\
\hline 137 & $\begin{array}{l}\text { Russula } \\
\text { nigricans }\end{array}$ & $\begin{array}{l}\text { Blackening } \\
\text { russula }\end{array}$ & Austria & $\begin{array}{c}\text { State of } \\
\text { Styria }\end{array}$ & $\begin{array}{c}1987- \\
1989\end{array}$ & $\begin{array}{l}{ }^{137} \text { Cs was mostly in } \\
\text { cap and lamellae; } \\
\text { Found in a spruce } \\
\text { and fir forest soil; } \\
\text { near } 800 \mathrm{~m} \text { asl }\end{array}$ & $\begin{array}{c}2001 \text { to } 4000 \\
\mathrm{~Bq} / \mathrm{kg} \mathrm{ww}\end{array}$ & $\begin{array}{c}\mathrm{TF}: 0.5 \text { to } \\
1.0\end{array}$ & Heinrich (1993) \\
\hline 137 & $\begin{array}{c}\text { Russula } \\
\text { olchroleuca }\end{array}$ & $\begin{array}{l}\text { Yellow-ocher } \\
\text { russula }\end{array}$ & Germany & $\begin{array}{l}\text { Southern } \\
\text { Bavaria }\end{array}$ & $\begin{array}{c}1987- \\
1990\end{array}$ & $\begin{array}{l}\text { In fruiting body } \\
\text { (assumed) }\end{array}$ & $\begin{array}{c}\text { Ratioed to } B \text {. } \\
\text { badius } * *: 0.2 \\
1 \text { to } 2.70 \\
1480 \text { to } 3870 \\
\text { Bq } / \mathrm{kg} \mathrm{fw} \\
\end{array}$ & $\begin{array}{c}\text { TF: } 0.53 \text { to } \\
1.8 ; \\
\text { Mean: } 1.1\end{array}$ & $\begin{array}{l}\text { Kammerer et al. } \\
\text { (1994) }\end{array}$ \\
\hline 137 & $\begin{array}{c}\text { Russula } \\
\text { olchroleuca }\end{array}$ & $\begin{array}{l}\text { Yellow-ocher } \\
\text { russula }\end{array}$ & $\begin{array}{c}\text { United } \\
\text { Kingdom }\end{array}$ & $\begin{array}{l}\text { Lady Wood } \\
\text { Forest, } 0.5 \\
\text { km from } \\
\text { Sellafield }\end{array}$ & $\begin{array}{l}\text { Sum- } \\
\text { mer } \\
1996\end{array}$ & $\begin{array}{l}\text { In fruiting body; } \\
\text { Cleaned before } \\
\text { analyses; From a } \\
\text { Picea sitchensis } \\
\text { woodland } \\
\end{array}$ & $\begin{array}{l}112 \text { to } 1558 \\
\mathrm{~Bq} / \mathrm{kg} \\
\text { Mean: } 633 \\
\mathrm{~Bq} / \mathrm{kg}\end{array}$ & $\begin{array}{l}\mathrm{T}_{\mathrm{ag}}: 0.014 \\
\text { to } 0.06\end{array}$ & Toal et al. (2002) \\
\hline 137 & $\begin{array}{c}\text { Russula } \\
\text { olchroleuca }\end{array}$ & $\begin{array}{l}\text { Yellow-ocher } \\
\text { russula }\end{array}$ & $\begin{array}{c}\text { United } \\
\text { Kingdom }\end{array}$ & $\begin{array}{l}\text { Linn Park } \\
\text { and Dollar }\end{array}$ & $\begin{array}{c}1987 \\
1990\end{array}$ & $\begin{array}{l}\text { In fruiting body; } \\
\text { Ectomycorrhizal } \\
\text { species }\end{array}$ & $195.2 \mathrm{~Bq} / \mathrm{kg}$ & No data & Watling et al. (1993) \\
\hline 137 & $\begin{array}{c}\text { Russula } \\
\text { olchroleuca }\end{array}$ & $\begin{array}{l}\text { Yellow-ocher } \\
\text { russula }\end{array}$ & Austria & $\begin{array}{l}\text { State of } \\
\text { Styria }\end{array}$ & $\begin{array}{c}1987- \\
1989\end{array}$ & $\begin{array}{l}{ }^{137} \text { Cs was mostly in } \\
\text { cap and lamellae; } \\
\text { Found in a spruce } \\
\text { and fir forest soil; }\end{array}$ & $\begin{array}{c}2001 \text { to } 4000 \\
\mathrm{~Bq} / \mathrm{kg} \mathrm{ww}\end{array}$ & $\begin{array}{c}\mathrm{TF}: 0.25 \text { to } \\
0.5\end{array}$ & Heinrich (1993) \\
\hline
\end{tabular}


WSRC-MS-2006-0422 Rev. 1

\begin{tabular}{|c|c|c|c|c|c|c|c|c|c|}
\hline & & & & & & 500 to $1000 \mathrm{~m}$ asl & & & \\
\hline 137 & $\begin{array}{c}\text { Russula } \\
\text { xerampelina }\end{array}$ & Crab russula & Germany & $\begin{array}{l}\text { Southern } \\
\text { Bavaria }\end{array}$ & $\begin{array}{c}1987- \\
1990\end{array}$ & $\begin{array}{l}\text { In fruiting body } \\
\text { (assumed) }\end{array}$ & $\begin{array}{c}\text { Ratioed to } B \text {. } \\
\text { badiu**:0.29 } \\
\text { to } 1.16 ; 620 \\
\text { to } 5175 \\
\mathrm{~Bq} / \mathrm{kg} \mathrm{fw} \\
\end{array}$ & $\begin{array}{l}\text { TF: } 0.83 \text { to } \\
5.4 ; \\
\text { Mean: } 2.0\end{array}$ & $\begin{array}{l}\text { Kammerer et al. } \\
\text { (1994) }\end{array}$ \\
\hline 137 & $\begin{array}{l}\text { Russula } \\
\text { sardonia }\end{array}$ & $\begin{array}{l}\text { Changeable } \\
\text { or pungent } \\
\text { russula }\end{array}$ & $\begin{array}{l}\text { United } \\
\text { Kingdom }\end{array}$ & $\begin{array}{l}\text { Lady Wood } \\
\text { Forest, } 0.5 \\
\text { km from } \\
\text { Sellafield }\end{array}$ & $\begin{array}{l}\text { Sum- } \\
\text { mer } \\
1996\end{array}$ & $\begin{array}{l}\text { In fruiting body; } \\
\text { Cleaned before } \\
\text { analyses; From a } \\
\text { Picea sitchensis } \\
\text { woodland }\end{array}$ & $1558 \mathrm{~Bq} / \mathrm{kg}$ & $\mathrm{T}_{\mathrm{ag}}: 0.06$ & Toal et al. (2002) \\
\hline 137 & $\begin{array}{l}\text { Sarcodon } \\
\text { aspratus }\end{array}$ & & Japan & Iwate & $\begin{array}{l}\text { Sept.- } \\
\text { Dec. } \\
1990\end{array}$ & $\begin{array}{l}\text { All portions; Cleaned } \\
\text { before analyses; } \\
\text { Edible species; } \\
\text { Mycorrhizal fungi } \\
\end{array}$ & $2080 \mathrm{~Bq} / \mathrm{kg}$ & No data & $\begin{array}{l}\text { Yoshida et al. } \\
\text { (1994); Yoshida and } \\
\text { Muramatsu (1994) }\end{array}$ \\
\hline 134 & $\begin{array}{l}\text { Sarcodon } \\
\text { aspratus }\end{array}$ & & Japan & Iwate & $\begin{array}{l}\text { Sept.- } \\
\text { Dec. } \\
1990\end{array}$ & $\begin{array}{c}\text { All portions; Cleaned } \\
\text { before analyses; } \\
\text { Edible species; } \\
\text { Mycorrhizal fungi } \\
\end{array}$ & $16.1 \mathrm{~Bq} / \mathrm{kg}$ & No data & $\begin{array}{l}\text { Yoshida et al. } \\
\text { (1994); Yoshida and } \\
\text { Muramatsu (1994) }\end{array}$ \\
\hline 137 & $\begin{array}{l}\text { Sarcodon } \\
\text { imbricatus }\end{array}$ & $\begin{array}{l}\text { Blue-footed } \\
\text { Scaly tooth or } \\
\text { Scaly hydnum } \\
\end{array}$ & Ukraine & $\begin{array}{l}\text { Zhitomir } \\
\text { region }\end{array}$ & $\begin{array}{c}1996- \\
1998\end{array}$ & $\begin{array}{l}\text { In fruiting body; } \\
\text { From Scots pine and } \\
\text { birch forests }\end{array}$ & $\begin{array}{c}\text { Mean:97900 } \\
\mathrm{Bq} / \mathrm{kg}\end{array}$ & TF:22.2 & $\begin{array}{l}\text { Vinichuk and } \\
\text { Johanson (2003) }\end{array}$ \\
\hline 137 & $\begin{array}{l}\text { Sarcodon } \\
\text { imbricatus }\end{array}$ & $\begin{array}{l}\text { Blue-footed } \\
\text { Scaly tooth or } \\
\text { Scaly hydnum }\end{array}$ & Germany & $\begin{array}{l}\text { Southern } \\
\text { Bavaria }\end{array}$ & $\begin{array}{c}1987- \\
1990\end{array}$ & $\begin{array}{l}\text { In fruiting body } \\
\text { (assumed) }\end{array}$ & $\begin{array}{l}\text { Ratioed to } B . \\
\text { badius**:0.2 } \\
9 \text { to } 1.16 ; \\
410 \text { to } 1170 \\
\text { Bq/kg fw }\end{array}$ & $\begin{array}{c}\text { TF:0.1 to } \\
1.2 ; \\
\text { Mean:0.59 }\end{array}$ & $\begin{array}{l}\text { Kammerer et al. } \\
\text { (1994) }\end{array}$ \\
\hline 137 & $\begin{array}{l}\text { Sarcodon } \\
\text { imbricatus }\end{array}$ & $\begin{array}{l}\text { Blue-footed } \\
\text { Scaly tooth or } \\
\text { Scaly hydnum }\end{array}$ & Austria & $\begin{array}{l}\text { State of } \\
\text { Styria }\end{array}$ & $\begin{array}{c}1987- \\
1989\end{array}$ & $\begin{array}{l}{ }^{137} \text { Cs was mostly in } \\
\text { cap and lamellae; } \\
\text { Found in a spruce } \\
\text { and fir forest soil; } \\
800 \mathrm{~m} \text { asl }\end{array}$ & $\begin{array}{c}501 \text { to } 1000 \\
\mathrm{~Bq} / \mathrm{kg} \mathrm{ww}\end{array}$ & $\begin{array}{c}\mathrm{TF}: 0.25 \text { to } \\
2.0\end{array}$ & Heinrich (1993) \\
\hline 137 & $\begin{array}{l}\text { Scleroderma } \\
\text { citrinum }\end{array}$ & Earthball & $\begin{array}{l}\text { United } \\
\text { Kingdom }\end{array}$ & $\begin{array}{l}\text { Balfour } \\
\text { Estate }\end{array}$ & $\begin{array}{c}1987 \\
1990\end{array}$ & $\begin{array}{l}\text { In fruiting body; } \\
\text { Ectomycorrhizal } \\
\text { species }\end{array}$ & $497.6 \mathrm{~Bq} / \mathrm{kg}$ & No data & Watling et al. (1993) \\
\hline 137 & $\begin{array}{l}\text { Suillus } \\
\text { bovinus }\end{array}$ & $\begin{array}{c}\text { Euro cow } \\
\text { bolete; } \\
\text { Shallow pored } \\
\text { fungus or } \\
\text { boletus } \\
\end{array}$ & Japan & $\begin{array}{l}\text { Akita, } \\
\text { Ibaraki and } \\
\text { Tochigi }\end{array}$ & $\begin{array}{l}\text { Sept.- } \\
\text { Dec. } \\
1990\end{array}$ & $\begin{array}{l}\text { All portions; Cleaned } \\
\text { before analyses; } \\
\text { Mycorrhizal fungi }\end{array}$ & $\begin{array}{c}77 \text { to } 1330 \\
\mathrm{~Bq} / \mathrm{kg}\end{array}$ & No data & $\begin{array}{l}\text { Yoshida et al. } \\
\text { (1994); Yoshida and } \\
\text { Muramatsu (1994) }\end{array}$ \\
\hline 134 & $\begin{array}{l}\text { Suillus } \\
\text { bovinus }\end{array}$ & $\begin{array}{c}\text { Euro cow } \\
\text { bolete; } \\
\text { Shallow pored } \\
\text { fungus or } \\
\text { boletus } \\
\end{array}$ & Japan & $\begin{array}{l}\text { Akita, } \\
\text { Ibaraki and } \\
\text { Tochigi }\end{array}$ & $\begin{array}{l}\text { Sept.- } \\
\text { Dec. } \\
1990\end{array}$ & $\begin{array}{l}\text { All portions; Cleaned } \\
\text { before analyses; } \\
\text { Mycorrhizal fungi }\end{array}$ & $<17 \mathrm{~Bq} / \mathrm{kg}$ & No data & $\begin{array}{l}\text { Yoshida et al. } \\
\text { (1994); Yoshida and } \\
\text { Muramatsu (1994) }\end{array}$ \\
\hline 137 & $\begin{array}{l}\text { Suillus } \\
\text { bovinus }\end{array}$ & $\begin{array}{c}\text { Euro cow } \\
\text { bolete; } \\
\text { Shallow pored } \\
\text { fungus or } \\
\text { boletus } \\
\end{array}$ & Germany & $\begin{array}{l}\text { Southern } \\
\text { Bavaria }\end{array}$ & $\begin{array}{c}1987- \\
1990\end{array}$ & $\begin{array}{l}\text { In fruiting body } \\
\text { (assumed) }\end{array}$ & $\begin{array}{c}\text { Ratioed to } B . \\
\text { badius } * *: 0.2 \\
7 \text { to } 0.4 ; 660 \\
\text { to } 760 \mathrm{~Bq} / \mathrm{kg} \\
\mathrm{fw} \\
\end{array}$ & $\begin{array}{l}\text { TF: } 0.35 \text { to } \\
0.98 ; \\
\text { Mean: } 1.0\end{array}$ & $\begin{array}{l}\text { Kammerer et al. } \\
(1994)\end{array}$ \\
\hline 137 & $\begin{array}{l}\text { Suillus (or } \\
\text { Boletus) } \\
\text { cavipes }\end{array}$ & $\begin{array}{c}\text { Hollow- } \\
\text { stemmed } \\
\text { boletus; } \\
\text { Hollow stalk }\end{array}$ & Italy & $\begin{array}{l}\text { Northeastern } \\
\text { Portion }\end{array}$ & 1986 & $\begin{array}{c}\text { All portions; After } \\
\text { Chernobyl; Grows on } \\
\text { acidic soils; } \\
\text { Saprophytic } \\
\end{array}$ & $\begin{array}{c}1460 \text { to } 5146 \\
\mathrm{~Bq} / \mathrm{kg}\end{array}$ & $\begin{array}{c}\mathrm{TF}: 0.146 \\
\text { to } 0.64\end{array}$ & $\begin{array}{l}\text { Battiston et al. } \\
(1989)\end{array}$ \\
\hline 137 & $\begin{array}{l}\text { Suillus (or } \\
\text { Boletus) } \\
\text { cavipes }\end{array}$ & $\begin{array}{l}\text { Hollow- } \\
\text { stemmed } \\
\text { boletus; } \\
\text { Hollow stalk }\end{array}$ & Italy & $\begin{array}{l}\text { Northeastern } \\
\text { Portion }\end{array}$ & 1986 & $\begin{array}{l}\text { All portions; Does } \\
\text { not include }{ }^{137} \mathrm{Cs} \\
\text { from Chernobyl; } \\
\text { Grows on acidic } \\
\text { soils; Saprophytic }\end{array}$ & $\begin{array}{c}389 \text { to } 1843 \\
\mathrm{~Bq} / \mathrm{kg}\end{array}$ & No data & $\begin{array}{l}\text { Battiston et al. } \\
(1989)\end{array}$ \\
\hline 134 & $\begin{array}{l}\text { Suillus (or } \\
\text { Boletus) }\end{array}$ & $\begin{array}{l}\text { Hollow- } \\
\text { stemmed }\end{array}$ & Italy & $\begin{array}{c}\text { Northeastern } \\
\text { Portion }\end{array}$ & 1986 & $\begin{array}{l}\text { All portions; Does } \\
\text { not include }{ }^{137} \mathrm{Cs}\end{array}$ & $\begin{array}{c}557 \text { to } 1738 \\
\mathrm{~Bq} / \mathrm{kg}\end{array}$ & TF:0.014 & \begin{tabular}{|l}
$\begin{array}{l}\text { Battiston et al. } \\
(1989)\end{array}$ \\
\end{tabular} \\
\hline
\end{tabular}




\begin{tabular}{|c|c|c|c|c|c|c|c|c|c|}
\hline & cavipes & $\begin{array}{c}\text { boletus; } \\
\text { Hollow stalk }\end{array}$ & & & & $\begin{array}{l}\text { from Chernobyl; } \\
\text { Grows on acidic } \\
\text { soils; Saprophytic }\end{array}$ & & to 0.44 & \\
\hline 137 & $\begin{array}{c}\text { Suillus } \\
\text { granulatus }\end{array}$ & $\begin{array}{l}\text { Dotted-stalk } \\
\text { bolete }\end{array}$ & Ukraine & $\begin{array}{l}\text { Zhitomir } \\
\text { Region }\end{array}$ & $\begin{array}{c}1996- \\
1998\end{array}$ & $\begin{array}{l}\text { In fruiting body; } \\
\text { From Scots pine and } \\
\text { birch forests }\end{array}$ & $\begin{array}{c}\text { Mean:44200 } \\
\mathrm{Bq} / \mathrm{kg}\end{array}$ & TF: 12.2 & $\begin{array}{l}\text { Vinichuk and } \\
\text { Johanson (2003) }\end{array}$ \\
\hline 137 & $\begin{array}{c}\text { Suillus } \\
\text { granulatus }\end{array}$ & $\begin{array}{l}\text { Dotted-stalk } \\
\text { bolete }\end{array}$ & Japan & Ibaraki & $\begin{array}{l}\text { Sept.- } \\
\text { Dec. } \\
1990\end{array}$ & $\begin{array}{c}\text { All portions; Cleaned } \\
\text { before analyses }\end{array}$ & $\begin{array}{c}136 \text { to } 1150 \\
\mathrm{~Bq} / \mathrm{kg}\end{array}$ & $\begin{array}{l}\text { TF: } 16(0 \text { to } \\
5 \mathrm{~cm} \text { of } \\
\text { soil })\end{array}$ & $\begin{array}{l}\text { Yoshida et al. } \\
\text { (1994); Muramatsu } \\
\text { et al. (1991); } \\
\text { Yoshida and } \\
\text { Muramatsu (1994) }\end{array}$ \\
\hline 134 & $\begin{array}{l}\text { Suillus } \\
\text { granulatus }\end{array}$ & $\begin{array}{l}\text { Dotted-stalk } \\
\text { bolete }\end{array}$ & Japan & Ibaraki & $\begin{array}{l}\text { Sept.- } \\
\text { Dec. } \\
1990\end{array}$ & $\begin{array}{l}\text { All portions; Cleaned } \\
\text { before analyses }\end{array}$ & $<13 \mathrm{~Bq} / \mathrm{kg}$ & No data & $\begin{array}{l}\text { Yoshida et al. } \\
\text { (1994); Muramatsu } \\
\text { et al. (1991); } \\
\text { Yoshida and } \\
\text { Muramatsu (1994) }\end{array}$ \\
\hline 137 & $\begin{array}{l}\text { Suillus } \\
\text { granulatus }\end{array}$ & $\begin{array}{l}\text { Dotted-stalk } \\
\text { bolete }\end{array}$ & Germany & $\begin{array}{l}\text { Southern } \\
\text { Bavaria }\end{array}$ & $\begin{array}{l}1987- \\
1990\end{array}$ & $\begin{array}{l}\text { In fruiting body } \\
\text { (assumed) }\end{array}$ & $\begin{array}{l}\text { Ratioed to } B \text {. } \\
\text { badius**:0.0 } \\
7 \text { to } 0.26 ; \\
595 \text { to } 1080 \\
\text { Bq } / \mathrm{kg} \mathrm{fw}\end{array}$ & $\begin{array}{l}\text { TF: } 0.05 \text { to } \\
0.53 ; \\
\text { Mean: } 0.29\end{array}$ & $\begin{array}{l}\text { Kammerer et al. } \\
(1994)\end{array}$ \\
\hline 137 & $\begin{array}{l}\text { Tricholoma } \\
\text { album }\end{array}$ & & $\begin{array}{l}\text { United } \\
\text { Kingdom }\end{array}$ & Rhosesmor & $\begin{array}{l}1987 \\
1990\end{array}$ & $\begin{array}{l}\text { In fruiting body; } \\
\text { Ectomycorrhizal } \\
\text { species }\end{array}$ & $\begin{array}{l}\text { Below } \\
\text { detection }\end{array}$ & No data & Watling et al. (1993) \\
\hline 137 & $\begin{array}{l}\text { Tricholoma } \\
\text { atrosquam- } \\
\quad \text { osum }\end{array}$ & $\begin{array}{l}\text { Dirty trich } \\
\text { complex }\end{array}$ & $\begin{array}{l}\text { United } \\
\text { Kingdom }\end{array}$ & Powmill & $\begin{array}{l}1987 \\
1990\end{array}$ & $\begin{array}{l}\text { In fruiting body; } \\
\text { Ectomycorrhizal } \\
\text { species }\end{array}$ & $732 \mathrm{~Bq} / \mathrm{kg}$ & No data & Watling et al. (1993) \\
\hline 137 & $\begin{array}{l}\text { Tricholoma } \\
\text { cingulatum }\end{array}$ & & $\begin{array}{l}\text { United } \\
\text { Kingdom }\end{array}$ & Saline & $\begin{array}{c}1987 \\
1990\end{array}$ & $\begin{array}{l}\text { In fruiting body; } \\
\text { Ectomycorrhizal } \\
\text { species }\end{array}$ & $\begin{array}{c}247.2 \text { to } \\
3500 \mathrm{~Bq} / \mathrm{kg}\end{array}$ & No data & Watling et al. (1993) \\
\hline 137 & $\begin{array}{l}\text { Tricholoma } \\
\text { flavovirens }\end{array}$ & $\begin{array}{c}\text { Firwood } \\
\text { agaric; Cava- } \\
\text { lier; Man-on- } \\
\text { horseback }\end{array}$ & Japan & Ibaraki & $\begin{array}{l}\text { Sept.- } \\
\text { Dec. } \\
1990\end{array}$ & $\begin{array}{c}\text { All portions; Cleaned } \\
\text { before analyses; } \\
\text { Mycorrhizal fungi }\end{array}$ & $3110 \mathrm{~Bq} / \mathrm{kg}$ & No data & $\begin{array}{l}\text { Yoshida et al. } \\
\text { (1994); Yoshida and } \\
\text { Muramatsu (1994) }\end{array}$ \\
\hline 134 & $\begin{array}{l}\text { Tricholoma } \\
\text { flavovirens }\end{array}$ & $\begin{array}{c}\text { Firwood } \\
\text { agaric; Cava- } \\
\text { lier; Man-on- } \\
\text { horseback }\end{array}$ & Japan & Ibaraki & $\begin{array}{l}\text { Sept.- } \\
\text { Dec. } \\
1990\end{array}$ & $\begin{array}{l}\text { All portions; Cleaned } \\
\text { before analyses; } \\
\text { Mycorrhizal fungi }\end{array}$ & $<65 \mathrm{~Bq} / \mathrm{kg}$ & No data & $\begin{array}{l}\text { Yoshida et al. } \\
\text { (1994); Yoshida and } \\
\text { Muramatsu (1994) }\end{array}$ \\
\hline 137 & $\begin{array}{c}\text { Tricholoma } \\
\text { pessandatum }\end{array}$ & $\begin{array}{c}\text { Golden orange } \\
\text { tricholoma }\end{array}$ & Spain & Muňoveros & $\begin{array}{c}\text { Not } \\
\text { known }\end{array}$ & In fruiting body & $122 \mathrm{~Bq} / \mathrm{kg}$ & No data & Baeza et al. (2004) \\
\hline 137 & $\begin{array}{l}\text { Tricholoma } \\
\text { portentosum }\end{array}$ & $\begin{array}{l}\text { Sticky gray } \\
\text { trich }\end{array}$ & Japan & Ibaraki & $\begin{array}{l}\text { Sept.- } \\
\text { Dec. } \\
1990 \\
\end{array}$ & $\begin{array}{c}\text { All portions; Cleaned } \\
\text { before analyses; } \\
\text { Mycorrhizal fungi }\end{array}$ & $424 \mathrm{~Bq} / \mathrm{kg}$ & No data & $\begin{array}{l}\text { Yoshida et al. } \\
\text { (1994); Yoshida and } \\
\text { Muramatsu (1994) }\end{array}$ \\
\hline 134 & $\begin{array}{l}\text { Tricholoma } \\
\text { portentosum }\end{array}$ & $\begin{array}{l}\text { Sticky gray } \\
\text { trich }\end{array}$ & Japan & Ibaraki & $\begin{array}{l}\text { Sept.- } \\
\text { Dec. } \\
1990\end{array}$ & $\begin{array}{l}\text { All portions; Cleaned } \\
\text { before analyses; } \\
\text { Mycorrhizal fungi }\end{array}$ & $<11 \mathrm{~Bq} / \mathrm{kg}$ & No data & $\begin{array}{l}\text { Yoshida et al. } \\
\text { (1994); Yoshida and } \\
\text { Muramatsu (1994) }\end{array}$ \\
\hline 137 & $\begin{array}{l}\text { Tricholoma } \\
\text { terreum }\end{array}$ & $\begin{array}{l}\text { Grey agaric; } \\
\text { Dark gray } \\
\text { trich }\end{array}$ & Spain & Muňoveros & $\begin{array}{c}\text { Not } \\
\text { known }\end{array}$ & In fruiting body & $49 \mathrm{~Bq} / \mathrm{kg}$ & No data & Baeza et al. (2004) \\
\hline 137 & $\begin{array}{l}\text { Tricholoma } \\
\text { terreum }\end{array}$ & $\begin{array}{l}\text { Grey agaric; } \\
\text { Dark gray } \\
\text { trich }\end{array}$ & Japan & Ibaraki & $\begin{array}{l}\text { Sept.- } \\
\text { Dec. } \\
1990\end{array}$ & $\begin{array}{c}\text { All portions; Cleaned } \\
\text { before analyses; } \\
\text { Mycorrhizal fungi }\end{array}$ & $602 \mathrm{~Bq} / \mathrm{kg}$ & No data & $\begin{array}{l}\text { Yoshida et al. } \\
\text { (1994); Yoshida and } \\
\text { Muramatsu (1994) }\end{array}$ \\
\hline 134 & $\begin{array}{l}\text { Tricholoma } \\
\text { terreum }\end{array}$ & $\begin{array}{l}\text { Grey agaric; } \\
\text { Dark gray } \\
\text { trich }\end{array}$ & Japan & Ibaraki & $\begin{array}{l}\text { Sept.- } \\
\text { Dec. } \\
1990\end{array}$ & $\begin{array}{c}\text { All portions; Cleaned } \\
\text { before analyses; } \\
\text { Mycorrhizal fungi }\end{array}$ & $<21 \mathrm{~Bq} / \mathrm{kg}$ & No data & $\begin{array}{l}\text { Yoshida et al. } \\
\text { (1994); Yoshida and } \\
\text { Muramatsu (1994) }\end{array}$ \\
\hline 137 & $\begin{array}{l}\text { Tylopilus } \\
\text { felleus }\end{array}$ & Bitter bolete & Germany & $\begin{array}{l}\text { Southern } \\
\text { Bavaria }\end{array}$ & $\begin{array}{c}1987- \\
1990\end{array}$ & $\begin{array}{l}\text { In fruiting body } \\
\text { (assumed) }\end{array}$ & $\begin{array}{c}\text { Ratioed to } B . \\
\text { badius**:0.3 } \\
5 \text { to } 1.57\end{array}$ & No data & $\begin{array}{l}\text { Kammerer et al. } \\
(1994)\end{array}$ \\
\hline 137 & $\begin{array}{l}\text { Tylopilus } \\
\text { felleus }\end{array}$ & Bitter bolete & Austria & $\begin{array}{l}\text { State of } \\
\text { Styria }\end{array}$ & $\begin{array}{c}1987- \\
1989\end{array}$ & $\begin{array}{l}{ }^{137} \text { Cs was mostly in } \\
\text { cap and lamellae; } \\
\text { Found in a spruce }\end{array}$ & $\begin{array}{c}4001 \text { to } 8000 \\
\mathrm{~Bq} / \mathrm{kg} \mathrm{ww}\end{array}$ & $\begin{array}{c}\text { TF: } 2.01 \text { to } \\
4.0\end{array}$ & Heinrich (1993) \\
\hline
\end{tabular}


WSRC-MS-2006-0422 Rev. 1

\begin{tabular}{|c|c|c|c|c|c|c|c|c|c|}
\hline & & & & & & $\begin{array}{l}\text { and fir forest soil; } \\
500 \text { to } 1000 \mathrm{~m} \text { asl }\end{array}$ & & & \\
\hline $\begin{array}{l}\text { Stable } \\
{ }^{133} \mathrm{Cs}\end{array}$ & $\begin{array}{c}\text { Boletus, } \\
\text { Laccaria, } \\
\text { Cantharellus, } \\
\text { Rozites cape- } \\
\text { rata, Lactarius } \\
\text { rufus,Tylo- } \\
\text { pilus felleus }\end{array}$ & Many names & $\begin{array}{c}\text { Czech } \\
\text { Republic }\end{array}$ & Forests & $\begin{array}{l}\text { Late } \\
1990 \text { 's, } \\
\text { Early } \\
2000\end{array}$ & $\begin{array}{c}\text { In fruiting body; All } \\
\text { of these species are } \\
\text { known to accumulate } \\
\text { Cs and } \mathrm{Rb}\end{array}$ & $\begin{array}{l}\text { No data } \\
\text { provided }\end{array}$ & No data & $\begin{array}{l}\text { As cited in Řanda } \\
\text { and Kučera (2004) }\end{array}$ \\
\hline 137 & $\begin{array}{l}\text { General } \\
\text { Basido- } \\
\text { mycetes }\end{array}$ & Not specified & Japan & Ibaraki & 1990 & $\begin{array}{c}\text { All portions; Cleaned } \\
\text { before analyses }\end{array}$ & $\begin{array}{c}2 \text { to } 1630 \\
\mathrm{~Bq} / \mathrm{kg} ; \mathrm{Mean} \\
\text { of } 483 \mathrm{~Bq} / \mathrm{kg}\end{array}$ & No data & Yoshida et al. (1994) \\
\hline 137 & $\begin{array}{l}\text { General } \\
\text { Basido- } \\
\text { mycetes }\end{array}$ & Not specified & Japan & Ibaraki & 1989 & $\begin{array}{c}\text { All portions; Cleaned } \\
\text { before analyses }\end{array}$ & $\begin{array}{l}3 \text { to } 152 \\
\text { Bq/kg; } \\
\text { Mean: } 120 \\
\text { Bq/kg } \\
\end{array}$ & No data & Yoshida et al. (1994) \\
\hline 137 & General & $\begin{array}{c}\text { Edible } \\
\text { mushrooms }\end{array}$ & $\begin{array}{l}\text { Former } \\
\text { USSR }\end{array}$ & $\begin{array}{c}\text { Dalata, } \\
\text { Ovruc region }\end{array}$ & 1990 & $\begin{array}{l}\text { For prepared edible } \\
\text { portions }\end{array}$ & $\begin{array}{l}609 \mathrm{~Bq} / \mathrm{kg} \\
\text { fw to } 20800 \\
\mathrm{~Bq} / \mathrm{kg} \mathrm{ww}\end{array}$ & No data & Cooper et al. (1992) \\
\hline 137 & General & $\begin{array}{c}\text { Edible } \\
\text { mushrooms }\end{array}$ & $\begin{array}{l}\text { Former } \\
\text { USSR }\end{array}$ & $\begin{array}{l}\text { Byelorussia, } \\
\text { Bragin } \\
\text { region } \\
\end{array}$ & 1990 & $\begin{array}{l}\text { For prepared edible } \\
\text { portions }\end{array}$ & $\begin{array}{c}1320 \mathrm{~Bq} / \mathrm{kg} \\
\mathrm{fw}\end{array}$ & No data & Cooper et al. (1992) \\
\hline 137 & General & $\begin{array}{c}\text { Edible } \\
\text { mushrooms }\end{array}$ & $\begin{array}{l}\text { Former } \\
\text { USSR }\end{array}$ & Savici & 1990 & $\begin{array}{c}\text { For prepared edible } \\
\text { portions }\end{array}$ & $\begin{array}{c}5260 \mathrm{~Bq} / \mathrm{kg} \\
\text { fw to } 131000 \\
\mathrm{~Bq} / \mathrm{kg} \mathrm{dw}\end{array}$ & No data & Cooper et al. (1992) \\
\hline
\end{tabular}

* All dry weight (dw) values are reported unless otherwise noted; fw: fresh weight, ww: wet weight.

** TF: Transfer Factor typically represents the amount of Cs in mushroom $(\mathrm{Bq} / \mathrm{kg}, \mathrm{dw})$ divided by the amount of $\mathrm{Cs}$ in soil or growth substrate $(\mathrm{Bq} / \mathrm{kg}, \mathrm{dw})$; the aggregated transfer factor or $\mathrm{T}_{\mathrm{ag}}$ $\left(\mathrm{m}^{2} / \mathrm{kg}\right.$ ):represents the amount of $\mathrm{Cs}$ in mushroom (in Bq/kg, $\mathrm{dw}$ ) divided by the amount of aerial deposition of radiocesium (in $\mathrm{Bq} / \mathrm{m}^{2}$ ) as in Toal et al. (2002). TF values calculated by Kammerer et al. (1994) are based on $(\mathrm{Bq} / \mathrm{kg}, \mathrm{fw})$ divided by the amount of $\mathrm{Cs}$ in O-horizon of soil $(\mathrm{Bq} / \mathrm{kg}, \mathrm{dw})$

*** Values in Kammerer et al. (1994) are expressed relative to the other mushroom B. badius because it was common at all sites and had a fairly consistent ${ }^{137} \mathrm{Cs}$ level.

asl: Above sea level. 